\title{
Flow Pattern Identification of Horizontal Two-Phase Refrigerant Flow Using Neural Networks
}

Abdeel J. Roman ${ }^{\mathrm{a}}$, Paul J. Kreitzer ${ }^{\mathrm{b}}$, Jamie S. Ervin ${ }^{\mathrm{c}, *}$, Michael S. Hanchak ${ }^{\mathrm{c}}$, and Larry W. Byrd ${ }^{\mathrm{a}}$

${ }^{a}$ Air Force Research Laboratory, Wright-Patterson AFB, OH 45433 USA

${ }^{\mathrm{b}} \mathrm{UES}$ Inc., Dayton, OH 45432 USA

${ }^{c}$ University of Dayton Research Institute, Dayton, OH 45469-0044 USA

\begin{abstract}
In this work, electrical capacitance tomography (ECT) and neural networks were used to automatically identify two-phase flow patterns for refrigerant R-134a flowing in a horizontal tube. In laboratory experiments, high-speed images were recorded for human classification of liquid-vapor flow patterns. The corresponding permittivity data obtained from tomograms was then used to train feedforward neural networks to recognize flow patterns. An objective was to determine which subsets of data derived from tomograms could be used as input data by a neural network to classify nine liquidvapor flow patterns. Another objective was to determine which subsets of input data provide high identification success when analyzed by a neural network. Transitional flow patterns associated with common horizontal flow patterns were considered. A unique feature of the current work was the use of the vertical center of mass coordinate in pattern classification. The highest classification success rates occurred using neural network input which included the probability density functions (in time) for both spatially averaged permittivity and center of mass location in addition to the four statistical moments (in time) for spatially averaged permittivity data. The combination of these input data resulted in an average success rate of $98.1 \%$ for nine flow patterns. In addition, $99 \%$ of the experimental runs were either correctly classified or misclassified by only one flow pattern.
\end{abstract}

Keywords: Electrical capacitance tomography, Neural network, Refrigerant

\section{Nomenclature}

$\begin{array}{ll}A_{i} & \text { Individual pixel area }\left[\mathrm{m}^{2}\right] \\ A_{T} & \text { Sum of all pixel areas }\left[\mathrm{m}^{2}\right] \\ D & \text { Tube diameter }[\mathrm{m}] \\ \mathrm{D}_{\mathrm{b}} & \text { Bubble diameter }[\mathrm{m}] \\ \text { ECT } & \text { Electrical capacitance tomography } \\ \mathrm{L} & \text { Length }[\mathrm{m}]\end{array}$

*Corresponding author. E-mail address: jervin1@udayton.edu 


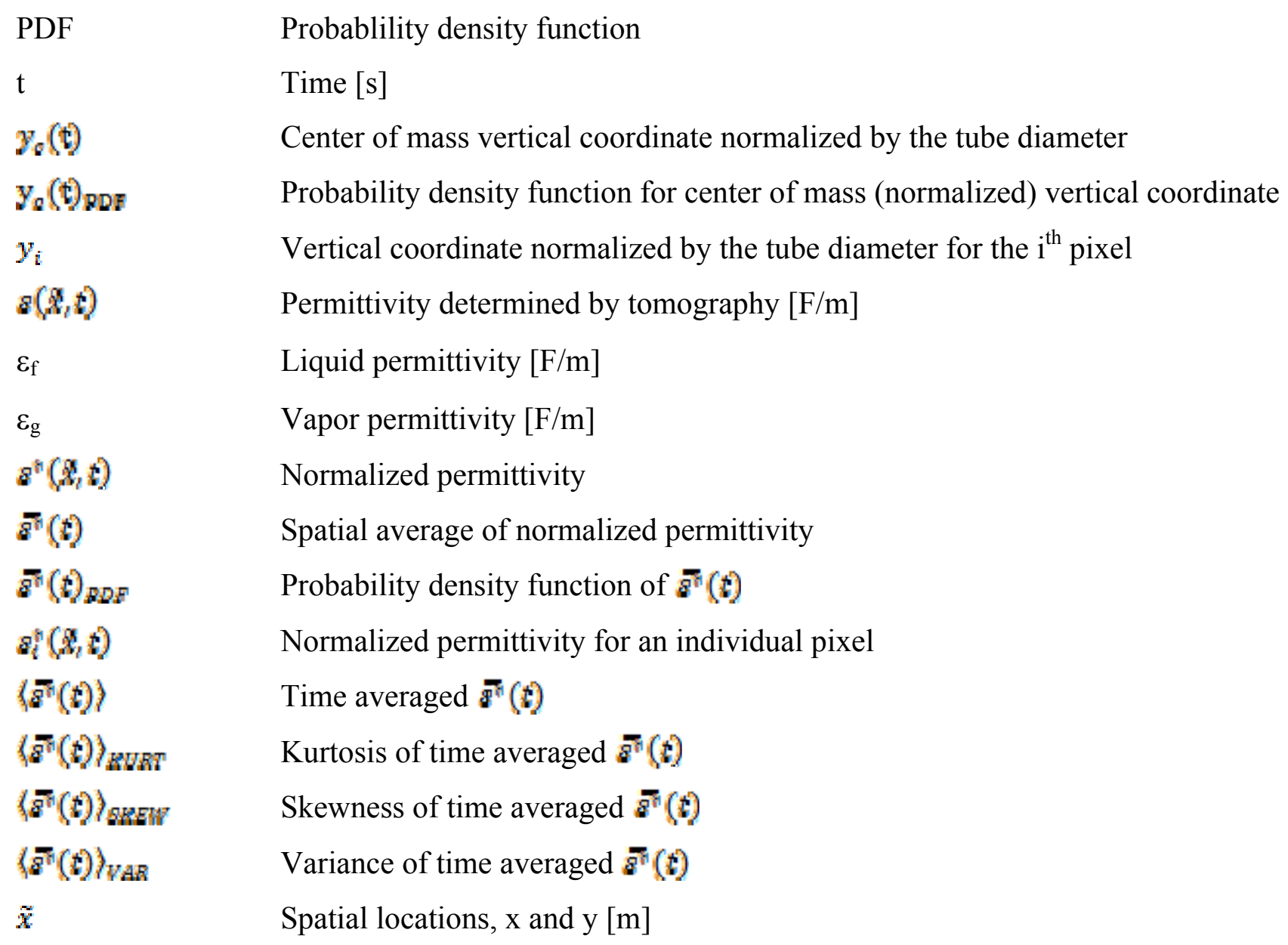

\section{Introduction}

Understanding the two-phase flow behavior of refrigerants is important for the design of advanced aircraft cooling systems. Specifically, the classification of liquid-vapor structures into flow patterns is useful for predicting heat transfer rates and, ultimately, system performance. Most flow and heat transfer correlations require a priori knowledge of the two-phase flow pattern and are based on steady-state conditions [1,2]. Although flow pattern identification can be performed using high-speed imaging, this method generally relies on the visual interpretation of liquid-vapor patterns. Unfortunately, visual interpretation can be highly subjective [2]. As a consequence, numerous flow pattern classifications have been defined in the past [3-7]. In contrast to visual observation, which is often impractical, nonvisual sensor signals can be analyzed to provide more objective classifications. Moreover, sensor signals are desired as inputs to real-time modeling and control.

Capacitance techniques are non-invasive and rely on differences in electrical permittivity to distinguish between liquid and vapor phases [2,8-9]. Capacitance measurements acquired 
simultaneously with high-speed videos of air-water flows have been used to identify two-phase flow patterns [2]. In addition, encouraging results involving the identification of two-phase flow patterns of refrigerants have been obtained using a capacitance probe that produced void fraction signals [10]. However, a single capacitance probe (as in $[2,10]$ ) may not fully characterize the liquid-vapor spatial distribution within a flow passage. This could lead to incorrect assumptions about the actual flow behavior. In contrast, electrical capacitance tomography (ECT) can provide a nearly instantaneous view of the liquid-vapor distribution within the system without optical access. Tomography may be used to derive the permittivity distribution from capacitance data [8-9]. The permittivity distribution, in turn, can provide the spatial distribution of the liquid and vapor phases. Many past research efforts involving ECT have focused on industrial applications where qualitative results were sufficient [8]. The use of ECT in detailed studies of liquid and vapor in horizontal flow has been limited, much less with the use of dielectric refrigerants which are of interest here $[8,10,11]$.

Artificial neural networks are used for pattern recognition and trend prediction involving complex processes. In an artificial neural network, the neurons (often called nodes) receive input signals, and each node calculates an individual output using a weighted sum and nonlinear activation function. Learning is achieved by the adjustment of these weights [12]. Past studies suggest that there is potential for using a neural network to objectively classify liquid-vapor distribution data [13-15]. These studies have used measured or simulated impedance (conductance) signals rather than input from ECT [13-16]. In addition, nearly all previous flow identification studies that have used neural networks were performed for vertical flows.

In this paper, the use of ECT together with neural networks to identify liquid-vapor flow patterns is explored. For this purpose, experiments involving the horizontal flow of refrigerant R-134a through a tube of small diameter $(7 \mathrm{~mm})$ were conducted. A horizontal-two phase flow can be categorized into one of several flow patterns which may include bubbly, plug, slug, stratified-wavy, and annular flows [17]. Here, previous work is extended by including additional transitional flow patterns corresponding to four of the above flow patterns for horizontal flow. The names of the transitional flow patterns are derived from these four flow patterns and are given here as the bubbly-transitional, plug-transitional, slugtransitional, and stratified-wavy-transitional patterns. In the current work, high-speed images of the flow patterns were recorded for purposes of initial human classification and final training verification. Processed permittivity data obtained from two-dimensional tomograms was used to train neural networks. A goal was to classify nine horizontal two-phase flow patterns with reasonable speed using 
neural networks as a predictive tool. Input information for the neural networks included the spatially averaged permittivity, center of mass location, and their probability density functions (in time). It also included four statistical moments (in time) for spatially averaged permittivity data. Another objective was to determine which subsets of input data provide high identification success for the flow patterns when analyzed by a neural network.

\section{Experimental Setup}

To explore the use of ECT in the identification of two-phase flow patterns, laboratory experiments were performed in which liquid-vapor flow patterns were generated for flow in a horizontal tube. Figure 1 shows a schematic of the experimental arrangement in which liquid R-134a was pumped through a heater to produce two-phase flow. To obtain different liquid-vapor flow patterns, the volumetric flow rates were adjusted in the range 0.1 to $0.5 \mathrm{~L} / \mathrm{min}$, while varying the heater power between 0 and $500 \mathrm{~W}$. Downstream from the heated section, the two-phase flow entered a fused quartz observation section (tube with $7 \mathrm{~mm}$ ID). The observation section permitted imaging using a high speed video camera (Phantom V4.2) and had thermocouples and pressure transducers located at $1.2 \mathrm{~m}$ increments along its length. The high speed camera was used to compare actual images of liquid-vapor flow with ECT characterization. R-134a passed through the cylindrical ECT sensor (ITS, 0690). Figure 1 shows one flow path for R-134a and another for cooling water which was used to condense R-134a vapor in the condenser (Lytron, LL510G02). The water was cooled by a chiller (PolyScience, 4260T).

Table 1 lists the measurement uncertainties associated with the thermocouples, pressure transducers (Omega PX-409), and flow meter (McMillan) shown in Figure 1. Experiments were performed with the refrigerant saturation conditions at the ambient temperature of $20{ }^{\circ} \mathrm{C}$. Relatively small temperature differences $\left(\sim 1{ }^{\circ} \mathrm{C}\right)$ between the ambient and refrigerant in the observation section of tubing provided reasonable grounds to neglect the heat transfer between them.

The ECT system consists of a multi-electrode sensor, electronics for capacitance determination, and data acquisition components [18]. The sensor fits around a section of the flow passage and measures changes in capacitance values between electrode pairs. Figure 2 a shows the configuration for eight electrodes (51 mm length), insulation, grounded screen, and measurement volume. Starting with electrode one, a voltage signal was applied to it. The capacitance was then measured from the remaining electrodes, two through eight. Next, electrode two became electrified, and electrodes three through eight were used to measure the capacitance. This process was repeated around the sensor until all (28) pairs of measurements were completed. A single tomogram was generated from these 28 capacitance 
measurements. In addition, a sensing unit conditioned the sensor signals and transmitted them to a data acquisition system. Details of the hardware and experimental procedures are available elsewhere [19].

Each tomogram provided a two-dimensional representation of the permittivity obtained from capacitance values at each sample time. Since the internal sensor volume encompassed a portion (51 $\mathrm{mm}$ ) of the flow path, the two-dimensional permittivity values were calculated from a three-dimensional measurement volume and averaged in time. Thus, the sensor length along the flow path as well as the sampling rate were practical limits for the sensor resolution. At the sampling rate of $\sim 48 \mathrm{~Hz}, 20 \mathrm{~ms}$ was necessary to acquire the data necessary for one two-dimensional tomogram with a delay of $1 \mathrm{~ms}$ between data sets. In this period, a two-phase feature with a speed of $\sim 1 \mathrm{~m} / \mathrm{s}$ would be detected by the sensor two times before leaving the sensing area. In the present experiments, the greatest velocity of a vapor feature was $\sim 0.85 \mathrm{~m} / \mathrm{s}$. Thus, the sampling rate was reasonable.

Equation 1 below was used to normalize the permittivity, $g(\{, t)$, that was determined by tomographic reconstruction. Here, $\tilde{x}$ represents the spatial variables, $\mathrm{x}$ and $\mathrm{y}$, and $\mathrm{t}$ is time. This normalization assigns $\varepsilon^{n}\left(X_{t}\right)$ to be zero for only vapor $\left(\varepsilon_{\mathrm{g}}\right)$ and one for liquid $\left(\varepsilon_{\mathrm{f}}\right)$ alone. Since nonlinear electric field responses may generate values outside the normalized range, a baseline reference value $\left(\varepsilon_{\mathrm{f}}\right.$ or $\left.\varepsilon_{\mathrm{g}}\right)$ was recorded while the sensor contained only liquid $\left(\varepsilon_{\mathrm{f}}\right)$ or only vapor $\left(\varepsilon_{\mathrm{g}}\right)$. A tomogram may have regions that are assigned values of $g^{n}(\alpha)$ between zero and one since a given pixel may represent a liquid-vapor mixture.

$$
g^{n}(x, t)=\left(a(x, t)-a_{g}\right) /\left(a_{f}-a_{g}\right)
$$

In this work, the linear back projection algorithm was used in the software provided by the instrument manufacturer for the tomographic reconstruction. This two-dimensional (cross-sectional) representation is proportional to $s^{n}(\mathbb{S})$ within the sensor volume [18]. There are 812 pixels which comprise the computational domain for the reconstruction process (Figure 2b). Since the cross-sectional area is circular, some elements were outside the circular boundary and not used. In addition, sensitivity maps generated by the manufacturer were used to linearize the measured capacitance values which were ultimately used in the reconstruction algorithm [18]. Subsequent processing of the tomograms was performed and is described in the next section of this paper.

Table 2 lists the flow patterns, heating rates, volumetric flow rates (at the pump outlet), pressure range, tube diameter, and time duration for each recorded flow pattern case. The experiments were conducted by holding the volumetric flow rate constant and incrementally increasing the heater power (Figure 3). The heater power was held at a given value until the liquid-vapor behavior was steady or 
steady-periodic. Visual observations of the liquid-vapor behavior were made while recording ECT data. A total of 546 heater power and volumetric flow rate combinations were examined.

\section{Data Processing and Analysis}

Tomography was used to obtain $g^{n}(\mathscr{S}, t)$ from the capacitance measurements which, in turn, provided the two-dimensional distribution of the liquid and vapor phases within the section of tubing encompassed by the sensor. One issue concerning the tomographic data was to determine an acceptable number of input data to train the neural network in a reasonable time period (less than 10 minutes on a Dell Precision T7500 workstation, 64 bit dual processor). The required computational time was prohibitively large for directly using all 812 pixel values from each tomogram (500 tomograms were recorded for each run) for each of the 546 experimental runs as input data ( $812 \times 500 \times 546 \sim 2.2 \times 10^{8}$ data points) to train a neural network. It was proposed that a combination of spatial- and time- averaging together with other quantities might allow for rapid training of accurate neural networks. Here, we explore the use of the following as input data for neural network flow pattern identification: the spatial

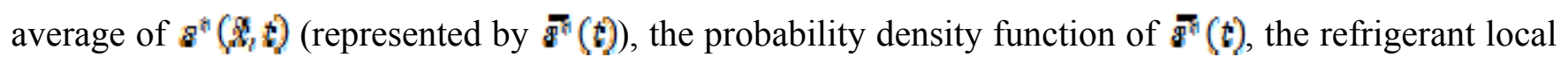
center of mass (i.e., the vertical coordinate normalized by the tube diameter and denoted by $y_{\varepsilon}(\mathrm{t})$ ), and the probability density function of $y_{a}(\mathrm{t})$. In addition, the time average of $\vec{g}^{n}(t)$ and the associated variance, kurtosis, and skewness values are considered.

$\vec{\Delta}(t)$ is given by Equation 2, where $A_{i}$ is the individual pixel area (Figure $\left.2 b\right), g_{i}^{n}(x)$ is the normalized permittivity for an individual pixel, $\mathrm{A}_{\mathrm{T}}$ is the sum of all (812) pixel areas, and $\mathrm{t}$ is time.

$$
\vec{s}(t)=\frac{1}{A_{T}} \sum_{i=1}^{812} a_{i}^{n}(x, t) A_{i}
$$

$\mathrm{A}_{\mathrm{i}}$ in Equation 2 is identical for all pixels and, thus, $\bar{s}(\mathrm{~s})$ was obtained by taking the arithmetic mean of the $g_{i}^{n}(\mathbb{S}, t)$ values for each tomogram at a particular time, t. Since $g^{n}\left(\mathscr{X}_{l}, t\right)$ is unity for the presence of liquid alone and zero for vapor alone, $\vec{s}(t)$ represents the time-varying liquid fraction in the sensor volume. Consequently, $1-\overline{g^{n}}(t)$ represents the time-varying void fraction.

For each flow pattern test point (i.e., each heater power and volumetric flow rate combination), 500 tomograms were generated over a sampling period of $30 \mathrm{~s}$. The probability density function (PDF) provided a way to observe the range of $\vec{s}(t)$ occurring with time for a given case. Here, the PDF of

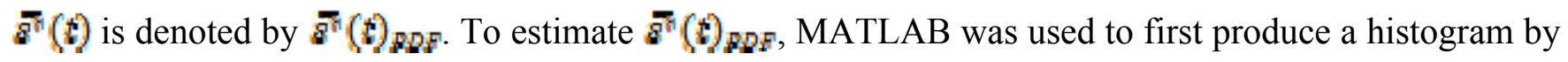
separating the 500 values of $\overrightarrow{F^{n}}(t)$ (obtained from the 500 tomograms) into bins [20]. Preliminary work showed that calculated histograms were sensitive to bin sizes below 25 but were insensitive to those in 
the range 25-100. Thus, 50 bins were used for determining all histograms. The estimate of $\bar{s}(t)$ was then generated by a smooth fit to the histogram. In addition, MATLAB was used to estimate the time

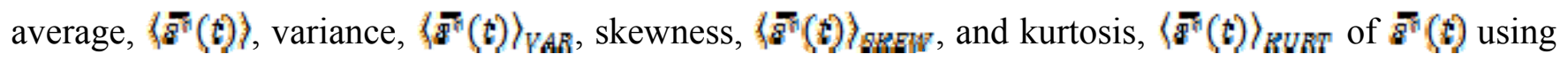
values from the 500 tomograms. $\left\langle\overrightarrow{F^{n}}(t)\right\rangle$ provides a representative value for the time-varying void

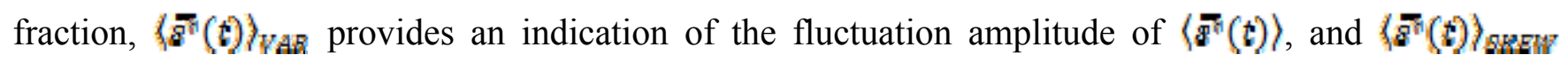
provides a measure of the asymmetry of the probability density function. In terms of a physical interpretation, a time series that has numerous intermittent extreme events would have a relatively high $\langle\bar{s}(t)\rangle_{\text {WWRT }}$. Thus, a high kurtosis would, for example, be associated with the rapidly alternating occurrence of large vapor bubbles and liquid.

A running average was used to estimate the number of data samples required to obtain a stable time average [21]. The running average of $\bar{\nabla}(t)$ was used to determine an acceptable number of data points to estimate $\langle\bar{\nabla}(t)\rangle$. When the fluctuations in the running average were within a $\pm 5 \%$ band of the stable average, the time average was assumed to be stable and the number of data points sufficient.

Since the vertical distribution of the liquid and vapor phases in a horizontal flow can be influenced by gravity, it may be advantageous to use the vertical coordinate of the center of mass, $y_{\odot}(t)$, of the tomogram (Figure $2 b$ ) as a neural network input. Since the origin of the coordinate system is at the center of the tube, $y_{\varepsilon}(t)$ is zero for a single phase flow within the tube. Because the positive direction for the y axis is up with respect to the gravity vector, a two-phase flow pattern will result in negative $y_{\theta}(t)$ values due to gravity drawing liquid to the bottom of the tube. $a_{i}\left(x_{i}\right)$ and the densities of the saturated vapor $\left(\rho_{g}\right)$ and liquid $\left(\rho_{f}\right)$ were used to estimate the fluid mass associated with a given pixel for the center of mass calculation as given by equation (3). In equation (3), $y_{i}$ represents a vertical pixel coordinate that is normalized by the tube diameter.

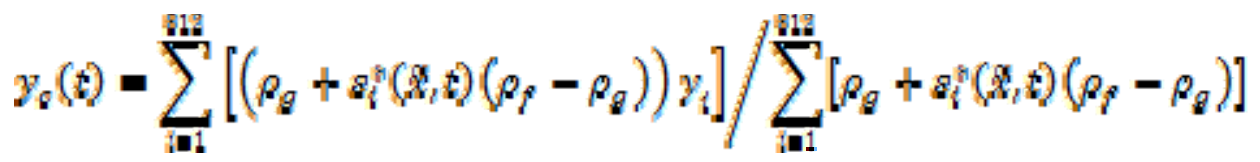

Following the calculation procedure with $\overline{\Omega^{-}}(t)$, PDFs for $y_{8}(t)$ were generated and are referred to as $y_{0}(t)$ PDF.

In this work, the MATLAB Neural Network package was used as the computational tool for identifying flow patterns. A feedforward neural network was selected for use due to its simplicity and ability to generalize to new situations [20]. This particular feedforward neural network had a hidden layer with nodes that used a sigmoid transfer function [20]. Training of a neural network was performed 
to identify the constants that comprise the weights and biases of each neuron. The number of hidden nodes was varied with different training sessions to determine an optimum number which would permit rapid calculations while avoiding overfitting. In this work, 50 was the maximum number of hidden nodes used. A single output layer was generated as a floating point number and rounded to the nearest integer value, corresponding to a flow pattern prediction.

The feedforward network together with the Levenberg-Marquardt training algorithm provide rapid calculation speed and are known for successful pattern identification [20]. Training was terminated when the generalization no longer improved which was indicated by an increase in the mean square error. The neural network was retrained by supplying it with additional randomized subsets of the original input and target data. This was repeated until the neural network predicted the observed flow patterns with an acceptable level of success, defined here to be the correct identification of flow patterns in at least $90 \%$ of the testing data. In the present work, the neural network was retrained up to twentyfive times to reach an acceptable level of success. The data used for testing were not part of the network training and, thus, provided an independent indication of neural network performance. That is, how well the network generalized beyond the supplied data [20]. Data from 546 experiments was randomly selected such that $30 \%$ were used for the validation and testing of a neural network, and $70 \%$ were used for training.

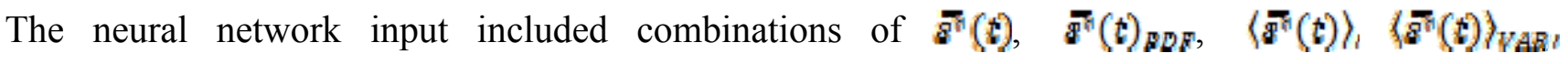

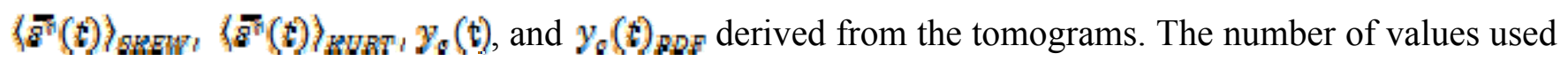
for each neural network input depended on the type of data itself. The number of values used for $\overline{\boldsymbol{s}}(\boldsymbol{D})$, $\bar{g}(t)_{\text {PDF }}, y_{0}(t)$, and $y_{\Omega}(t)_{D D F}$ are described in the next section. The quantities

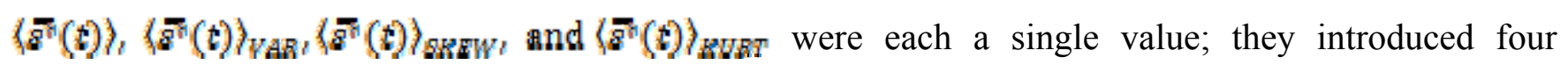
values for network training for each data set.

\section{Results and Discussion}

In this section, the flow patterns are first characterized by high speed imaging. This is followed by a characterization of flow patterns by $\overline{s^{n}}(t), \bar{s}(t)_{P D F}, y_{Q}(t)$, and $y_{Q}(t)$ DDF. The performance of the neural network inputs in classifying two-phase flow patterns is then described.

\subsection{High Speed Imaging}

High speed imaging was used for the initial human classification of the flow patterns. Table 3 shows representative images (view perpendicular to the tube axis) and cross-sectional drawings (view along the tube axis) for each flow pattern. It is useful to examine the images of Table 3 together with the plot in 
Figure 3, which shows the dependence of the flow pattern on both the input heater power and volumetric flow rate. Generally, there were clear visual demarcations among the flow patterns. However, overlapping liquid-vapor behavior existed due to the stochastic nature of the transition patterns. Care was taken to label the transitions correctly, or, in the case of great uncertainty, eliminate them altogether. Lastly, Table 3 lists the time-averaged void fraction range for each flow pattern. As described earlier, the void fraction estimate is given by $1-\langle\bar{a}(t))$.

Table 3 shows a representative image of bubbly flow. Bubbly flow was the first flow pattern encountered after single-phase liquid flow as the heater power was increased from zero with the volumetric flow rate at the highest setting (Table 2, $0.5 \mathrm{~L} / \mathrm{min}$ ). As illustrated in Table 3 (drawing of view along tube axis), discrete bubbles with diameters $\left(D_{b}\right)$ smaller than the tube radius $(D / 2)$ moved along the upper tube surface [17]. As the heater power was further increased at a constant flow rate (Figure 3), bubbly flow evolved into what is termed bubbly transitional flow. The image of bubbly transitional flow in Table 3 is similar to that of bubbly flow but elongated bubbles move along the upper tube surface. The drawing along the tube axis indicates that $\mathrm{D}_{\mathrm{b}}$ in the bubbly-transitional pattern were smaller than $\mathrm{D} / 2$, but the bubble length (L) could approach 2D.

Plug flow occurred when small adjacent bubbles coalesced into larger bubbles separated by liquid regions [17]. In addition, $\mathrm{D}_{\mathrm{b}}$ continued to be smaller than $\mathrm{D}$, but $\mathrm{L}$ was several $\mathrm{D}$, and the bubble frequency increased significantly. Bubbles in plug-transitional flow (Table 3 ) have $\mathrm{L}$ and $\mathrm{D}_{\mathrm{b}}$ similar to those in plug flow. However, plug-transitional bubbles have an elongated front section followed by a tail of smaller diameter. Slug flow evolved from plug transitional flow where L approached 80D. The bubbles had a consistent bullet shape with a rounded nose and tail. Also, $\mathrm{D}_{\mathrm{b}}$ began to approach D. Liquid regions separated the vapor bubbles and often entrained small dispersed bubbles [17]. Slugtransitional flow is similar to slug flow in that the bubbles have a bullet shape with a short tail (not in image). However, L was greater than that for slug flow with smaller liquid regions.

Stratified wavy flow occurred at high heater powers (Figure 3). The image of stratified wavy flow shows that liquid slugs no longer occurred, and a vapor layer existed along the upper portion of the tube. Kelvin-Helmholtz instabilities are responsible for waves which propagated along the tube length, often contacting the upper tube surface $[17,22]$. As in stratified wavy flow, interfacial waves were also present in stratified wavy transitional flow. The waves were more frequent but their crests did not contact the upper tube surface. However, they rose up on the tube wall wetting the lateral surface [17, 22]. Table 3 shows an example of annular flow consisting of liquid flow enveloping a flowing vapor 
core with small waves along the liquid-vapor interface. Annular flow is believed to occur when the vapor velocity increases beyond that in stratified wavy transitional flow [17, 22]. The liquid layer is thicker at the bottom due to gravity and did not always extend all the way to the top.

\section{$4.2 \bar{\nabla}(t)$ and $\bar{\nabla}(t)_{\text {PDF }}$ Characteristics for Different Flow Patterns}

The flow patterns are now described in terms of $\bar{s}(t)$ and $\overline{g^{-}}(t)$ DDF. Figure 4 shows representative plots of $\bar{s}(t)$ with time together with $\bar{s}(t)$ for nine flow patterns in the order of increasing void fraction. The plots correspond to the images, flow rates and heater powers of Table 3. Bubbly flow

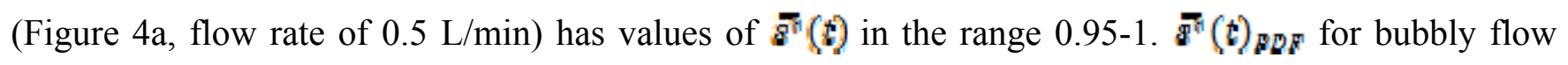
(Figure 4b) has a single peak near the upper end of the permittivity range, indicating that most of the flow is liquid. As the heater power increased at the flow rate of $0.5 \mathrm{~L} / \mathrm{min}$, bubbly transitional flow

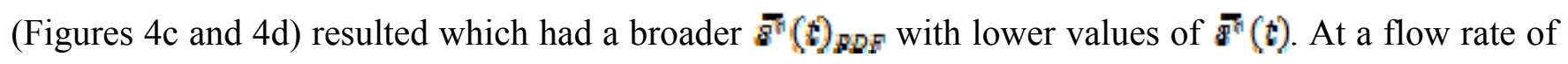
$0.3 \mathrm{~L} / \mathrm{min}$ and heater power near $30 \mathrm{~W}$ (Table 3 ), the $\bar{s}(\mathrm{~s})$ associated with plug flow (Figure 4e) have a nearly periodic behavior with values ranging from $0.56-0.98$. This nearly periodic behavior is confirmed by the image in Table 3 of flowing liquid plugs that are regularly separated by vapor. $\overline{F^{n}}\left(t_{A D F}\right.$ for plug flow (Figure 4f) has two distinguishable peaks for $\bar{s}(t)$ of 0.78 and 0.93 due to the presence of large volumes of alternating liquid and vapor phases. The liquid plugs appear less periodic in the plug transitional flow pattern (Figure $4 \mathrm{~g}$ ), and the corresponding $\overline{\boldsymbol{g}}(\mathbf{f})$ (Figure $4 \mathrm{~h}$ ) is similar although broader than $\overline{s^{n}}(t)$ for plug flow. Figure $4 \mathrm{i}$ shows that $\bar{g}^{n}(t)$ of the slug flow pattern has large, relatively rapid fluctuations that are associated with the passage of long vapor bubbles and short liquid slugs (Table 3 ). Figure $4 \mathrm{j}$ shows that the $\overline{g^{n}}()_{P D F}$ for slug flow, has a broad range. For slug transitional flow (Figure 4k), the heights of the peaks in $\bar{s}(t)$ have generally increased and the $\bar{s}(t)$ values in the $\overline{a n}()_{D D F}$ plot range from 0.21 to 0.98 (Figure 4l). Relative to slug flow, this indicates sequential vapor bubbles with larger volumes that are separated by a greater distances. The $\overline{\boldsymbol{s}^{n}}(\boldsymbol{t})$ associated with stratified wavy flow (Figure $4 \mathrm{~m}$ ) has large and small intermittent peaks relative to $\bar{s}(\boldsymbol{t})$ of the slug and slug transitional patterns. The intermittent peaks are due to interfacial waves which propagated along the tube length (Table 3 ). Relative to stratified wavy flow, Figure 4o shows that $\bar{s}^{n}(t)$ for stratified wavy transitional flow has significantly smaller peaks. In addition, Figure $4 \mathrm{p}$ shows that $\bar{F}(t){ }_{R D F}$ is entirely below 0.5 which also means that the void fraction was always greater than 0.5 . The amplitude of the fluctuations in $\bar{s}(\mathbf{s})$ for annular flow is small (Figure $4 q$ ) relative to those of stratified wavy or stratified wavy transitional flows. This is confirmed by the observation that interfacial waves were no longer 
visible (Table 3). In addition, the plot of $\bar{s}\left(t_{D D F}\right.$ for annular flow has a peak with no tail which accompanies a large void fraction (Table 3). This might be anticipated from the images of a flowing vapor core and liquid annulus within the tube (Table 3 ) for these flow patterns.

\section{3 $\mathrm{y}_{Q}(\mathrm{t})$, and $\mathrm{y}_{Q}(\mathrm{t})_{\mathrm{PDE}}$ Characteristics for Different Flow Patterns}

For a liquid-vapor flow that is horizontal with respect to gravity, the gas phase is near the upper surface of the flow passage with the liquid phase below if the tube is long enough for the vapor to stratify. Consequently, it is helpful to use the vertical coordinate of the tomogram centroid location as a neural network input. Figure 5 shows $\mathrm{y}_{g}(t)$, and $\mathrm{y}_{g}(t)_{\mathrm{PDF}}$ for each flow pattern. For reference, a ycoordinate value of 0 corresponds to the center of the tube. The peaks and troughs of $y_{q}(t)$ in Figure 5 generally track with those of $\bar{s}(t)$ in Figure 4 for the corresponding patterns. This occurs for two reasons. The first is that the center of mass shifts downward (upward) as the vapor fraction increases (decreases) due to the difference in phase densities in a gravity field. The second reason is that $\bar{s}(t)$ decreases with increasing vapor volume. Figures 4 and 5 show that $\bar{f}(t)_{D D F}$ and $y_{9}(t)_{P D F}$ both broaden beyond a single peak and then ultimately return to a single peak from bubbly to annular flows.

\subsection{Neural Network Studies}

It is useful to determine which combinations of input data types derived from ECT tomograms allow rapid, classification of two-phase flow patterns by a feedforward neural network. The current study was carried out in two stages with increasing complexity. In the first, bubbly, plug, slug, stratified-wavy, and annular patterns were considered since they are relatively well defined and have been treated elsewhere $[17,22]$. It was proposed that combinations of input data types that resulted in high success rates for classifying flow patterns in the first stage could then be used in the second, more challenging stage to identify nine flow patterns.

Table 4 compares the classification success rates for eight neural network input vectors for the five basic flow patterns used in the first stage of this study. The first column provides the flow pattern category, and the second lists the number of experimental runs for each flow pattern. The third through the tenth columns provide the classification success rates for input that was comprised of individual and combined data types. Here, the success rate is defined as the percentage of flow patterns predicted correctly. Based on past work involving vertical flows and other instrumentation, there was reason to believe that the use of neural network input values derived from time averaging or probability density

functions have potential for reasonable success rates $[13,14]$. Thus, the seventh column lists the success 


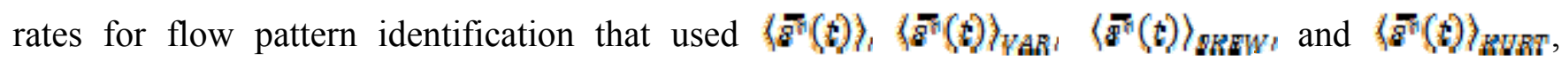
together as input for each experiment. Similarly, columns 8 through 10 list the success rates for input

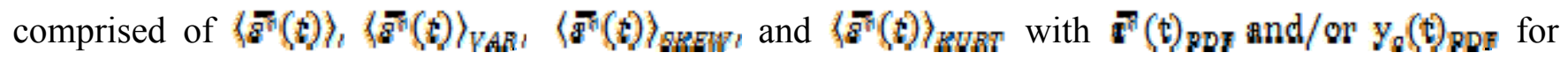
each experimental run. The second row in Table 4 provides the number of input data points for each data type. The bottom row lists the average success rate of the neural network resulting from the use of different input data types.

Table 4 shows that using either $\overrightarrow{s^{t}}(t)$ or $\mathrm{y}_{\mathrm{c}}(\mathrm{t})$ alone as an input resulted in the lowest overall success rates with the least success in defining slug flow. The use of other input types provided success rates greater than $90 \%$ for all flow patterns. Since a success rate of $90 \%$ or greater is a goal, Table 4 shows that it is acceptable to use any listed input other than $\bar{\nabla}(t)$ or $\mathrm{y}_{\mathrm{c}}(\mathrm{t})$ to identify the basic flow patterns. The average classification success rate over all patterns for columns 5 and 7 shows that the first four statistical (time) moments involving $\bar{s}(t)$ provide information that is only slightly better than that of $\bar{s}(t)_{\text {PDF }}$. This supports the conclusion of Rosa et al. [13] that the first four statistical (time) moments involving $\bar{s}(t)$ provide information essentially equivalent to that of $\vec{T}(t)$.

The bubbly, plug, slug, and stratified-wavy flow patterns were then subdivided into categories which included transition patterns. In these transition regions the flow may oscillate between the adjacent patterns making classification difficult. Consequently, Table 5 shows neural network classification results for nine flow patterns. The number of runs (second column) listed under the original and transition flow patterns in Table 5 sum to the original number of runs for the basic flow pattern in Table 4 (i.e., the transitional flow patterns in Table 5 are subsets of the basic flow patterns in Table 4). Lastly, the neural network input types of Table 5 are the same as those in Table 4 (i.e., columns 3-10).

Table 5 shows that the lowest success rates occurred using either $\bar{s}(t)$ or $y_{c}(t)$ alone as was shown in Table 4. In fact, the success rate using either as a network input was zero for bubbly and bubbly transitional flows. Zero success rates for $\bar{s}(t)$ and $y_{c}(t)$ are believed to be due to the challenge of differentiating among the strong similarities between bubbly and bubbly transitional patterns (Figures

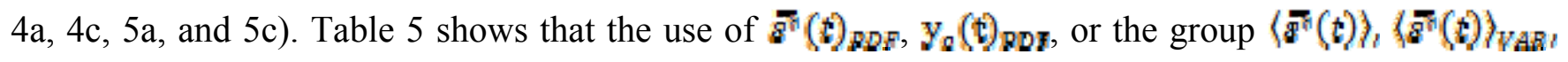

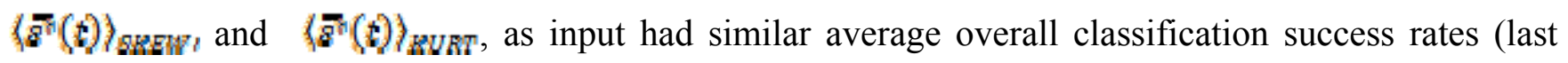
row) and offered significant improvement in classification success over that of $\bar{s}(t)$ or $y_{c}(t)$ for individual flow patterns. This can be attributed to more distinct differences among the PDFs and time averaged statistics for different patterns (PDFs in Figures 4-5 and, for example, the time averaged statistics as represented by the void fraction in Table 3, $1-\bar{s}(t)$. The bottom row of Table 5 shows that 
the average classification success rate over all flow patterns increases when the time averaged statistics

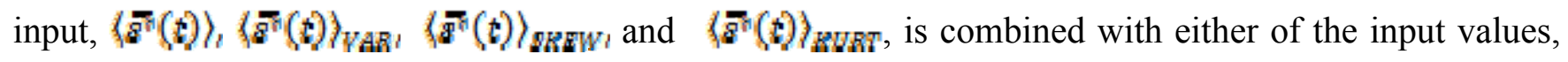
$\bar{s}(t)_{P D F}$ or $\mathrm{y}_{\Omega}(t)_{\mathrm{PDF}}$. Further, when $\bar{\nabla}(t)_{P D F}$ and $\mathrm{y}_{\Omega}(t)_{\mathrm{PDF}}$ are combined with time average statistics into a single input (column 10), classification success rates greater than $90 \%$ were obtained for all patterns. In addition, the average classification success rate over all flow patterns was $98.1 \%$.

The classification success rates for the above nine flow patterns obtained using the combination of time averaged statistics, $\overline{\vec{A}^{-}}(\mathrm{t})_{B D F}$, and $\mathrm{y}_{Q}(t)_{\mathrm{PDF}}$ (i.e., column 10) compare well, if not better, than those of past studies involving electrical capacitance measurements (ECT sensors) and horizontal flows. For example, Jeanmeure et al. [23] used an identification tree to classify horizontal flows but only used three patterns. Later, Yan et al. [24] used parameters obtained from steady, two-dimensional numerical simulations of nine flow patterns to train a back-propagation neural network. Their numerical simulations were correlated with experiments involving tomography but did not include the inherent unsteady nature of an actual two-phase flow. In addition, two of their patterns, a tube filled with liquid or empty, were relatively simplistic.

Table 5 lists the classification success rates for the flow patterns but does not specifically characterize

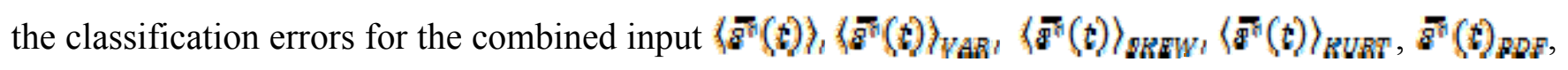
and $y_{Q}(t)_{R D F}$. An error value within \pm 0.5 of zero represents correct identification, otherwise a value outside these bounds is considered to be incorrect. For a total of 546 experiments, a small number (14) were incorrectly classified by one flow pattern and a much smaller number (1) were incorrectly classified by two patterns. More specifically, $99 \%$ of the experimental runs were either correctly classified or misclassified by no more than one flow pattern. Tables 4 and 5 together show that the input combination of time averaged statistics, $\overline{\vec{S}^{n}}(\mathrm{t})_{P D F}$, and $\mathrm{y}_{Q}(t)_{\mathrm{PDF}}$ can be used to reasonably classify flow patterns observed in horizontal flow.

\section{Conclusions}

This work investigated the use of ECT and neural networks to identify two-phase flow patterns for refrigerant R-134a flowing in a horizontal tube. Laboratory experiments were performed to generate different liquid-vapor flow patterns. High-speed images were recorded for human classification of the flow patterns. The corresponding permittivity data obtained from tomograms was used to train feedforward neural networks to recognize flow patterns. A goal was to classify nine horizontal twophase flow patterns using neural networks as a predictive tool. Another was to determine which subsets of input data provide high identification success for the flow patterns when analyzed by a neural 
network. Previous work involving horizontal flows was extended by considering transitional flow patterns associated with four common flow patterns (within a small-diameter tube). A unique feature of the current work is the use of the vertical center of mass coordinate in pattern classification for horizontal flow.

The highest classification success rate occurred with the use of neural network input consisting

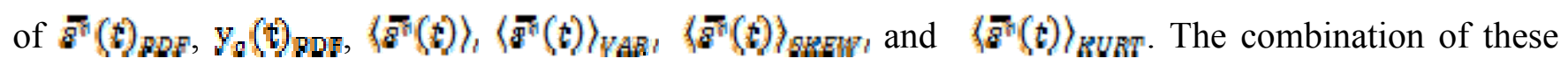
data resulted in a $100 \%$ success rate for the basic five flow patterns and a high average success rate of 98.1\% for nine flow patterns, which include the transitional patterns. In addition, $99 \%$ of the experimental runs were either correctly classified or misclassified by only one flow pattern. Based on the success rate of flow pattern identification with relatively small network training and run times, there is potential for the use of ECT combined with neural network analysis to automatically identify twophase patterns for horizontal flow in thermal process control applications.

\section{Acknowledgement}

This material is based on research sponsored by U.S. Air Force Contract No. FA8650-D-122224(TO0001). The authors are grateful for support from the Mechanical and Thermal Systems Branch of the Air Force Research Laboratory (AFRL/RQQM). The views and conclusions contained herein are those of the authors and should not be interpreted as necessarily representing the official policies or endorsements, either expressed or implied, of U.S. Air Force Office of Scientific Research or the U.S. Government.

\section{References}

[1]A.S. Dalkilic, S. Wongwises, Validation of void fraction models and correlations using a flow pattern transition mechanism model in relation to the identification of annular vertical downflow in-tube condensation of R134a, Int. Commun. Heat Mass, 37 (2010) 827-834.

[2] H. Caniere, C. T'Joen, A. Willockx, M. De Paepe, Capacitance signal analysis of horizontal twophase flow in a small tube, Exp. Therm. Fluid Sci., 32 (2008) 892-904.

[3] A. Ghajar, Non-boiling heat transfer in gas-liquid flow in pipes-a tutorial, J. of the Braz. Soc. of Mech. Sci. \& Eng., 27 (2005) 46-73.

[4] N.P. Ekberg, S.M. Ghiaasiaan, S.I. Abdel-Khalik, M. Yoda, S.M. Jeter, Gas-liquid two-phase flow in narrow horizontal annuli, Nucl. Eng. and Des., 192 (1999) 59-80.

[5] W. Qu, S.M. Yoon, I. Mudawar, Two-phase flow and heat transfer in rectangular micro-channels, J. Electron. Packaging, 126 (2004) 288-300. 
[6] J.M. Quiben, J.R. Thome, Flow pattern based on two-phase frictional pressure drop model for horizontal tubes Part II: new phenomenological model, Int. J. Heat Fluid Flow, 28 (2007) 1060-1072.

[7] K.A. Triplett, S.M. Ghiaasiaan, S.I. Abdel-Khalik, D.L. Sadowski, Gas-liquid two-phase flow in microchannels Part I: two-phase flow patterns, Int. J. Multiphase Flow, 25 (1999) 377-394.

[8] W. Warsito, L.S. Fan, Measurement of real-time flow structures in gas-liquid and gas-liquid-solid flow systems using electrical capacitance tomography (ECT), Chem. Eng. Sci., 56 (2001) 6455-6462.

[9] Z. Huang, B. Wang, H. Li, Application of electrical capacitance tomography to the void fraction measurement of two-phase flow, IEEE Trans. Instrum. Meas., 52 (2003) 7-12.

[10] H. Caniere, B. Bauwens, C. T'Joen, M. De Paepe, Mapping of horizontal refrigerant two-phase flow patterns based on clustering of capacitive sensor signals, Int. J. Heat Mass Transfer, 53 (2010) 5298-5307.

[11] E. dos Reis, L.Goldstein Jr., Characterization of slug flows in horizontal piping by signal analysis from a capacitive probe, Flow Meas. Instrum., 21 (2010) 347-355.

[12] T. Xie, S.M. Ghiaasiaan, S. Karrila, Artificial neural network approach for flow regime classification in gas-liquid-fiber flows based on frequency domain analysis of pressure signals, Chem. Eng. Sci., 59 (2004) 2241-2251.

[13] E.S. Rosa, R.M. Salgado, T. Ohishi, N. Mastelari, Performance comparison of artificial neural networks and expert systems applied to flow pattern identification in vertical ascendant gas-liquid flows, Int. J. Multiphase Flow, 36 (2010) 738-754.

[14] S. Gosh, D.K. Pratihar, B. Maiti, P.K. Das, Identification of flow regimes using conductivity probe signals and neural networks for counter-current gas-liquid two-phase flow, Chem. Eng. Sci., 84 (2012) 417-436.

[15] Y. Mi, M. Ishii, L.H. Tsoukalas, Flow regime identification methodology with neural networks and two-phase flow models, Nucl. Eng. and Des., 204 (2001) 87-100.

[16] H.L. Hu, J. Dong, J. Zhang, Y.J. Cheng, T.M. Xu, Identification of gas/solid two-phase flow regimes using electrostatic sensors and neural-network techniques, Flow Meas. Instrum., 22 (2011) 482487.

[17] J.G. Collier, J.R. Thome, Convective Boiling and Condensation, third ed., Oxford University Press, New York, 1994.

[18] Industrial Tomography Systems, Inc., ITS Tomography Toolsuite v7.0 Users Manual, April 2010. 
[19] P.J. Kreitzer, M. Hanchak, L. Byrd, Flow regime identification of horizontal two-phase refrigerant R-134a flow using neural networks, Paper IMECE2013-62510, IMECE, San Diego, CA, 2013.

[20] MATLAB r2010a, The MathWorks, Natick, MA.

[21] J.S. Bendat, A.G. Piersol, Random Data: Analysis and Measurement Procedures, Wiley, New York, 1971.

[22] Wolverine Tube Inc., Engineering Data Book III, pp. 12-3 to 12-11.

[23] L.F.C. Jeanmeure, T. Dyakowski, W.B.J. Zimmerman, W. Clark, Direct flow-pattern identification using electrical capacitance tomography, Exp. Therm. Fluid Sci., 26 (2002) 763-773.

[24] H. Yan, Y.H. Liu, C.T. Liu, Identification of flow regimes using back-propagation networks trained on simulated data based on a capacitance tomography sensor, Meas. Sci. Technol., 15 (2004) 432-436.

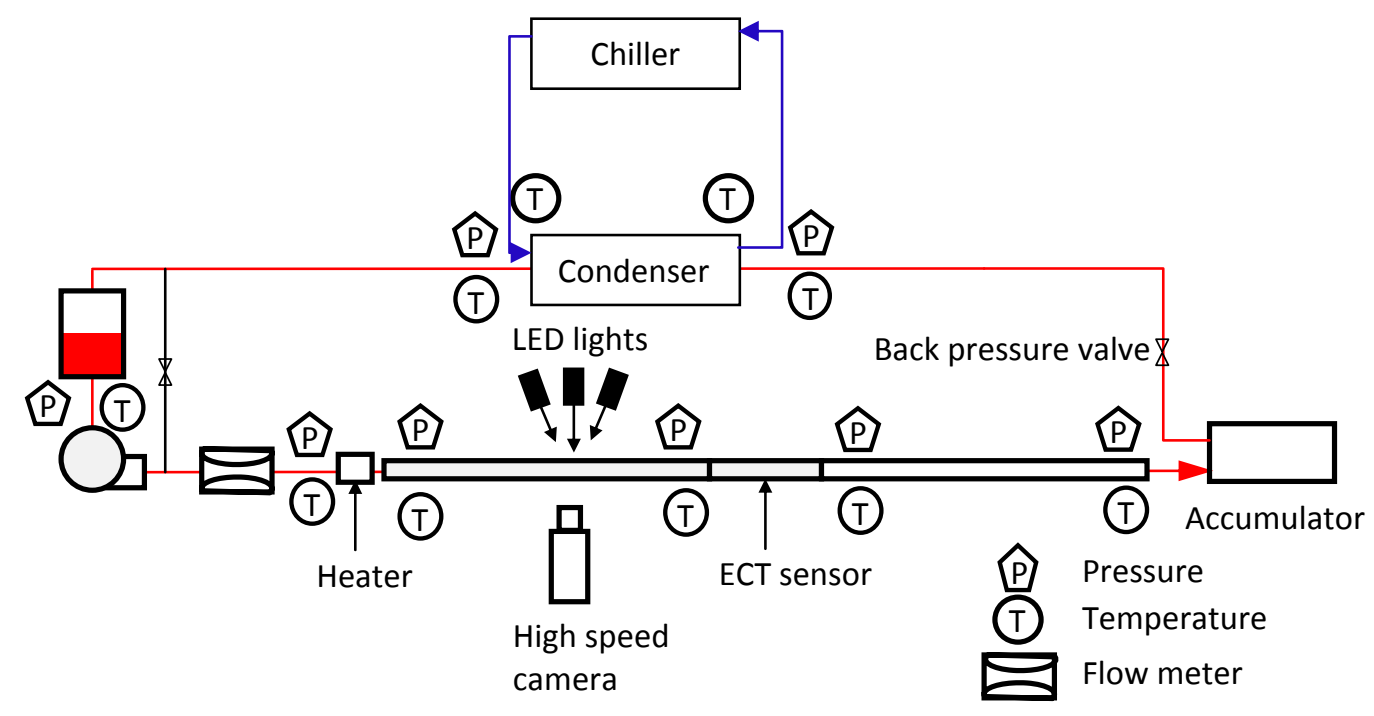

Figure 1. Schematic of two phase flow system. Red represents the refrigerant, and blue represents chilled water. 
a)

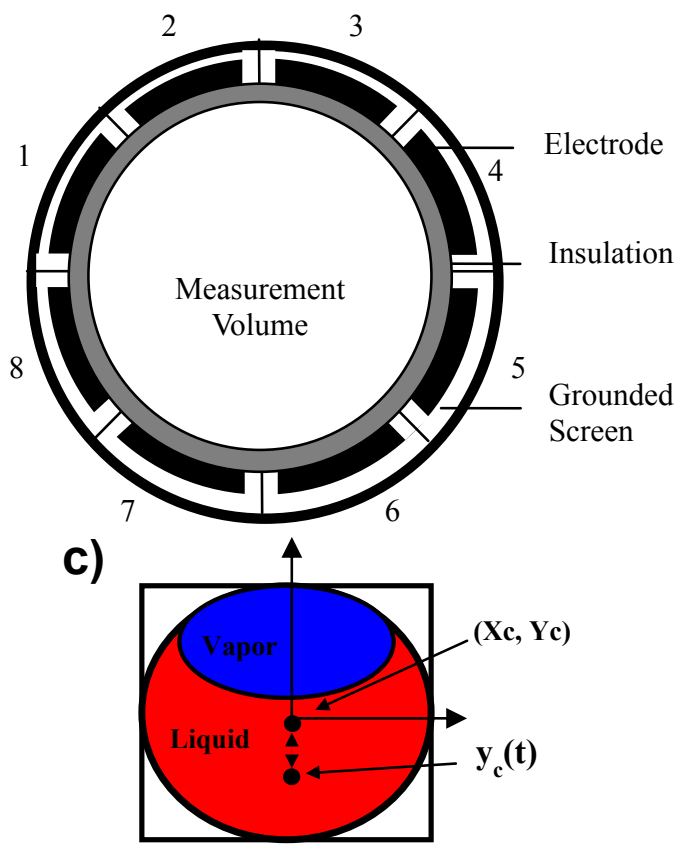

b)

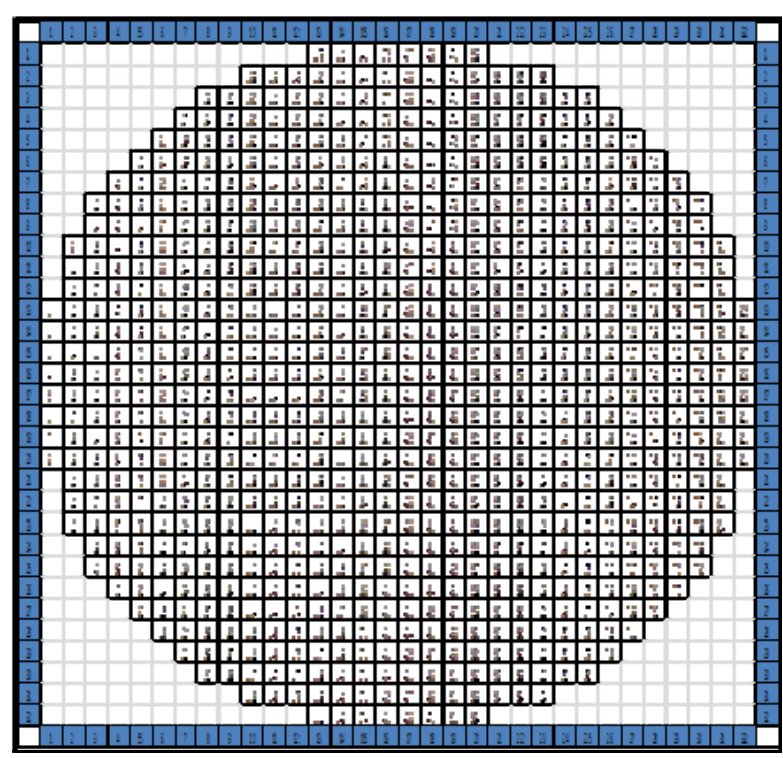

Figure 2. Drawings of the a) cross-sectional view of the sensor with eight electrodes, b) tomography computational domain consisting of 812 pixels, and c) instantaneous normalized vertical coordinate $\left(y_{c}(t)\right)$ corresponding to the refrigerant center of mass. The normalized coordinates $\left(X_{c}, Y_{c}\right)$ designate the origin.

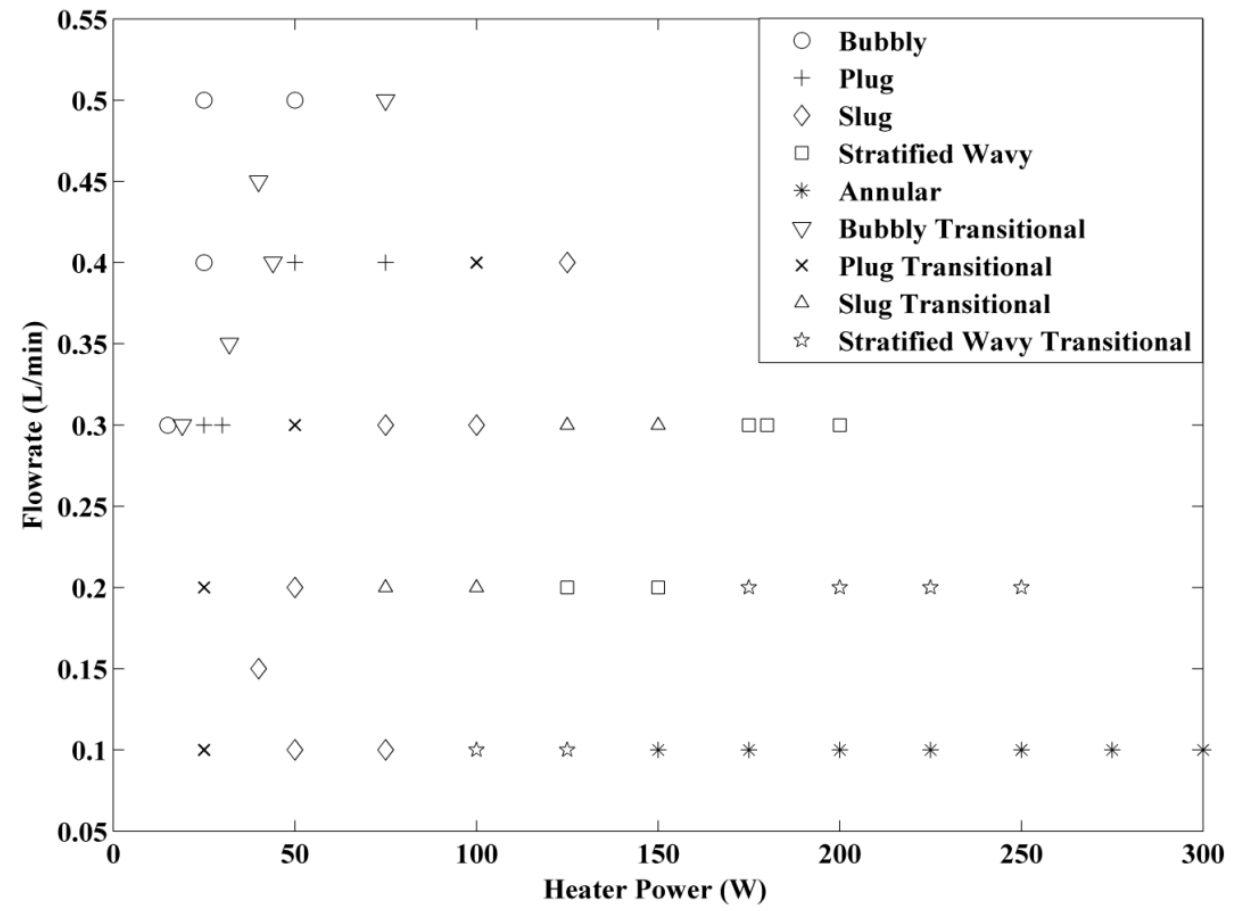

Figure 3. Visually observed flow patterns as a function of flow rate and heater power. 


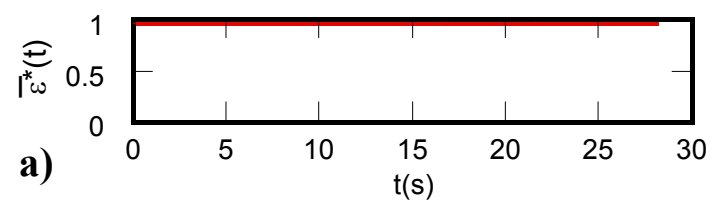

Bubbly

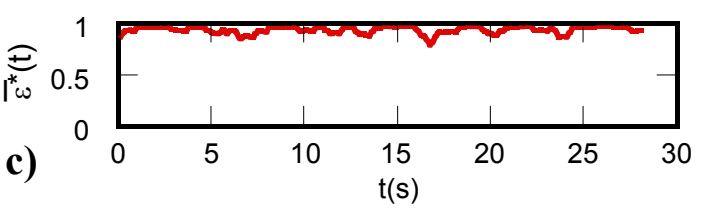

Bubbly

Transitional
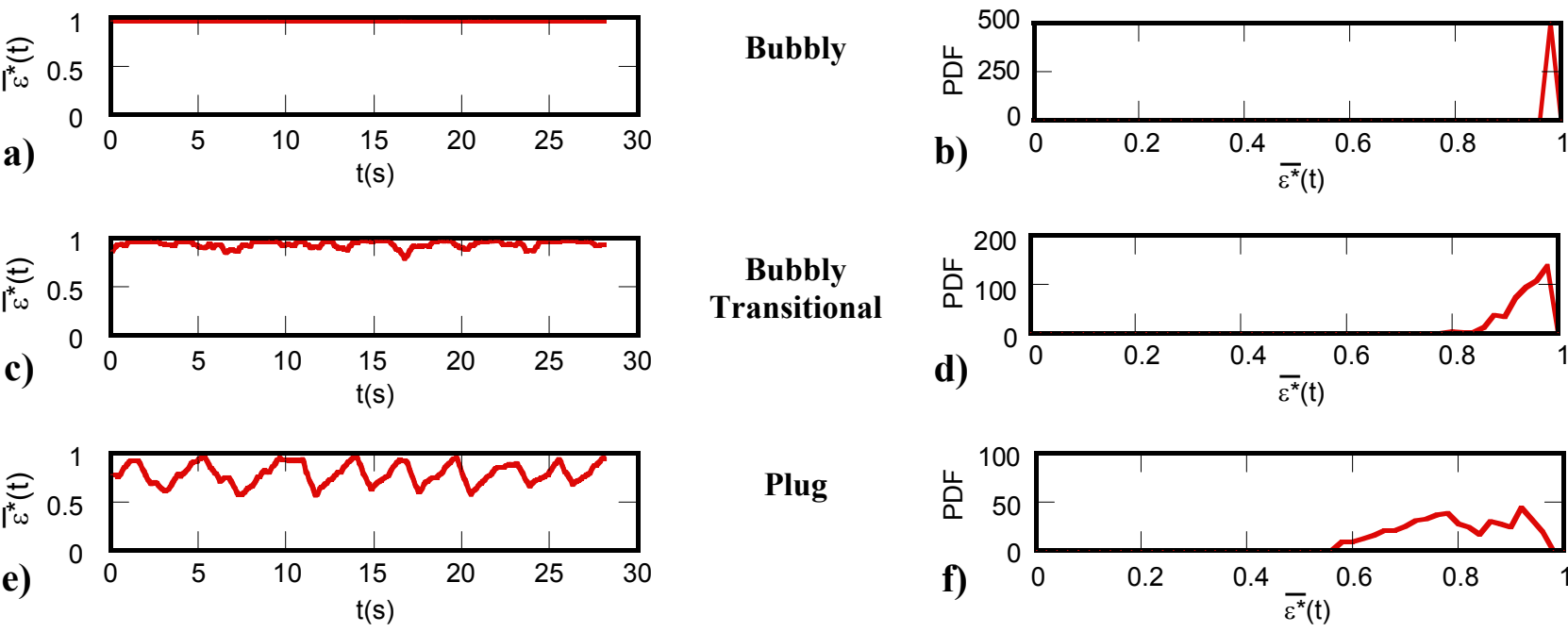

Plug
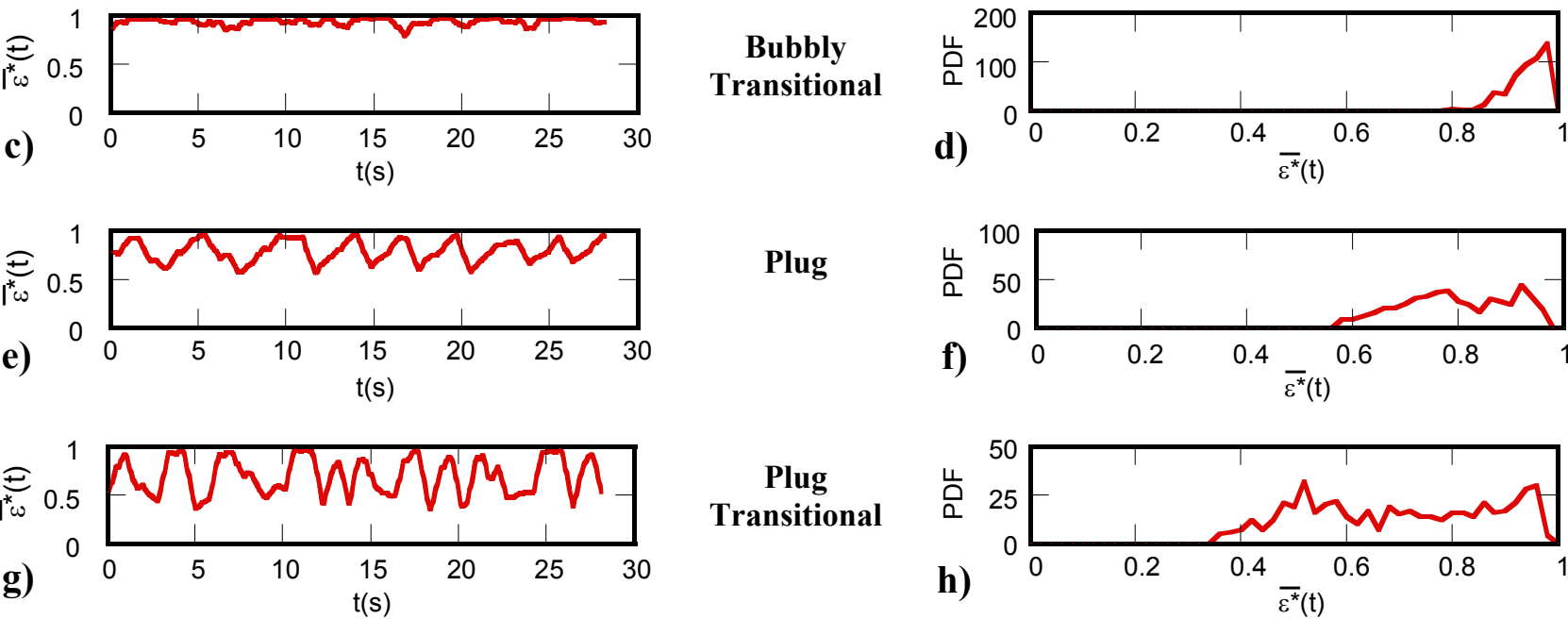

Plug

Transitional
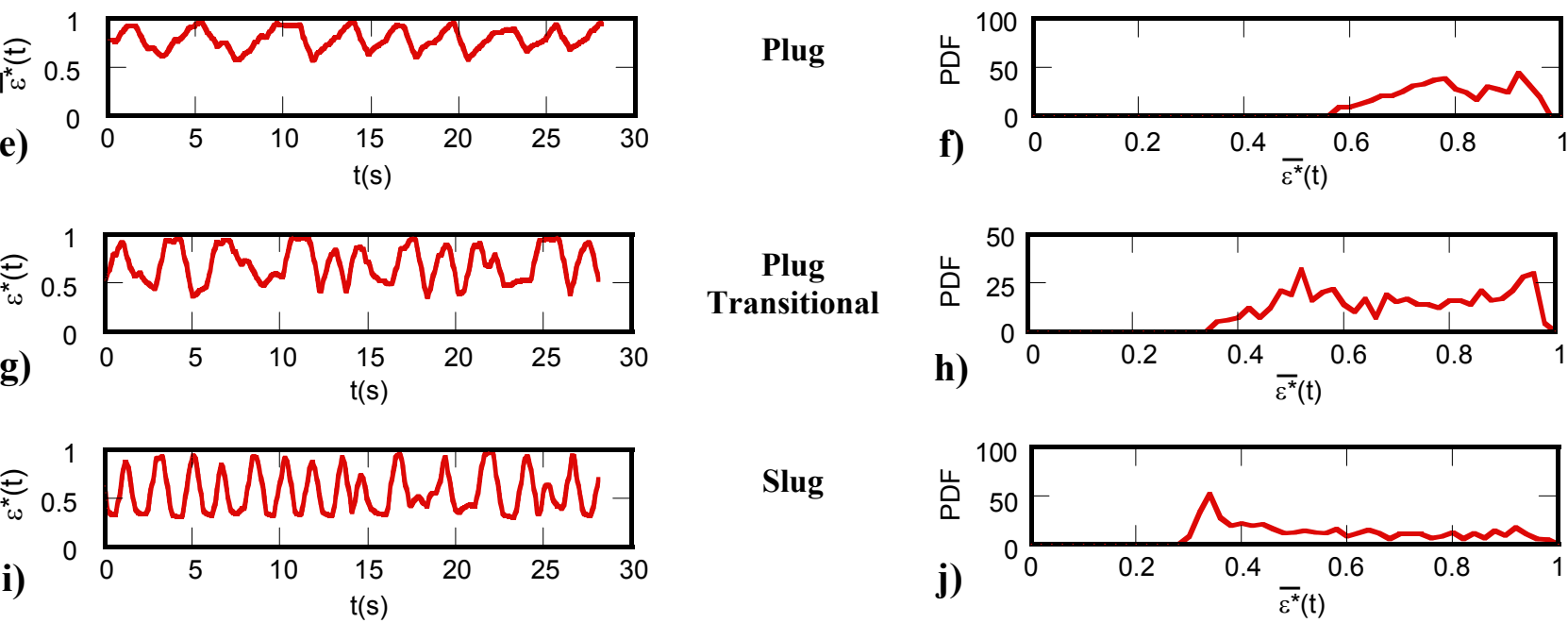

Slug
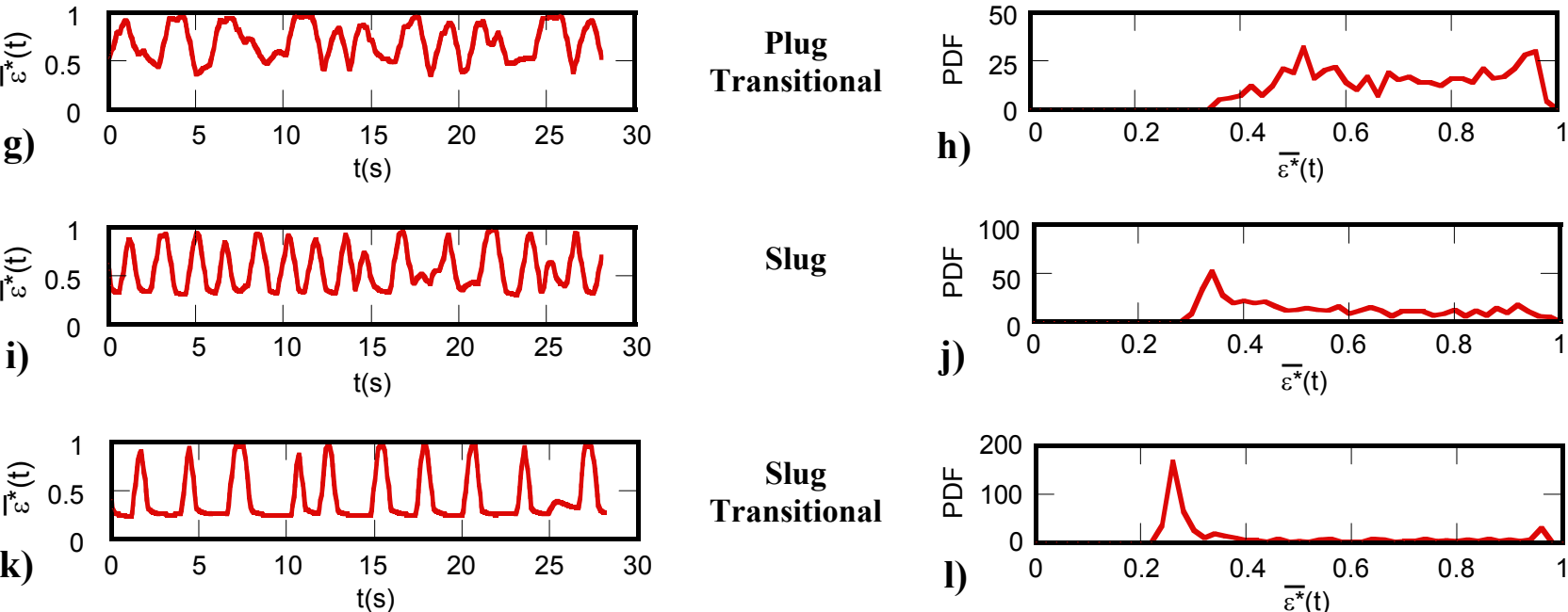

Slug

Transitional
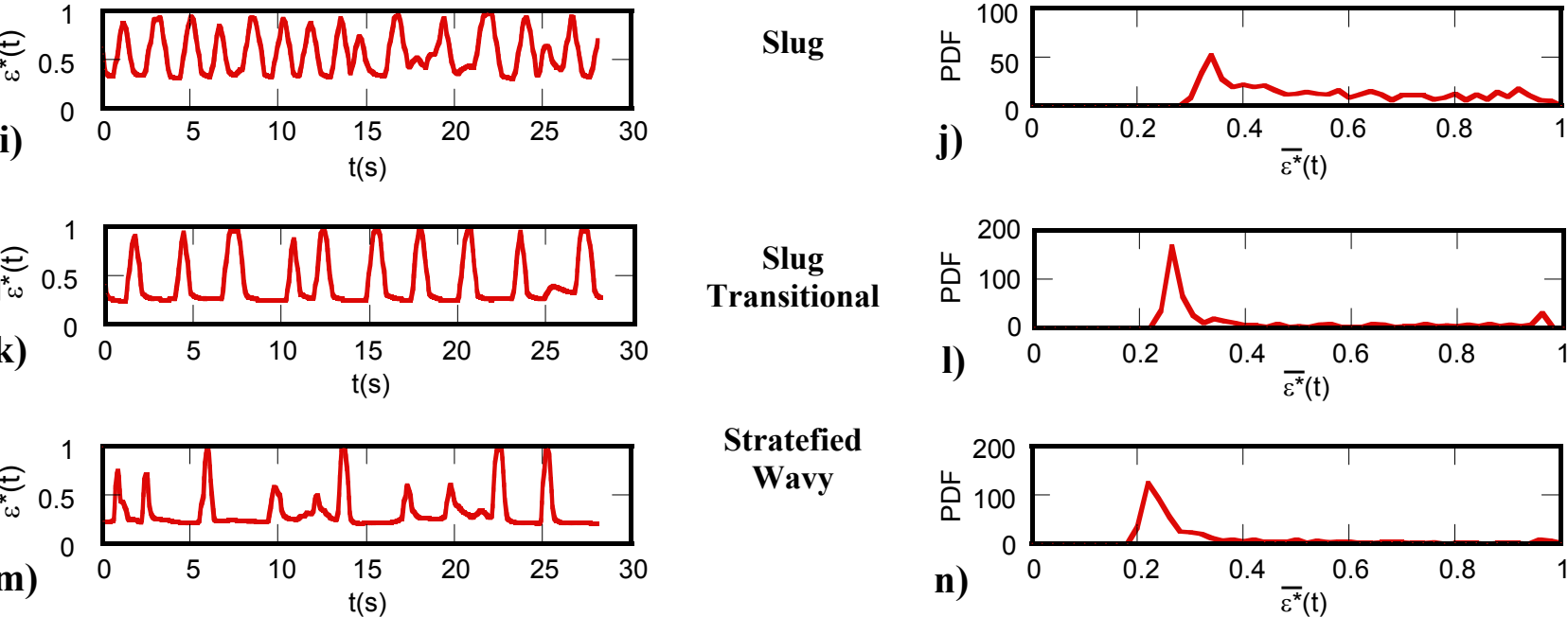

\section{Stratefied \\ Wavy}
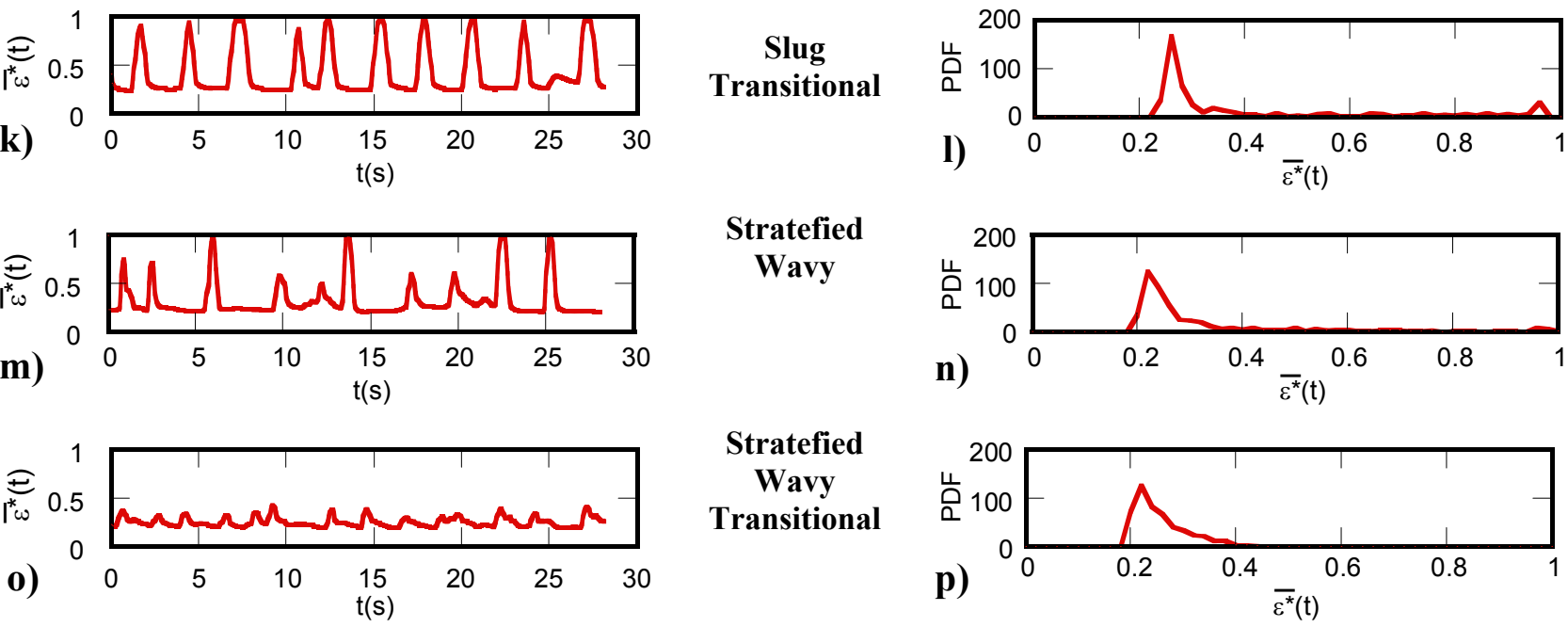

Stratefied

Wavy

Transitional
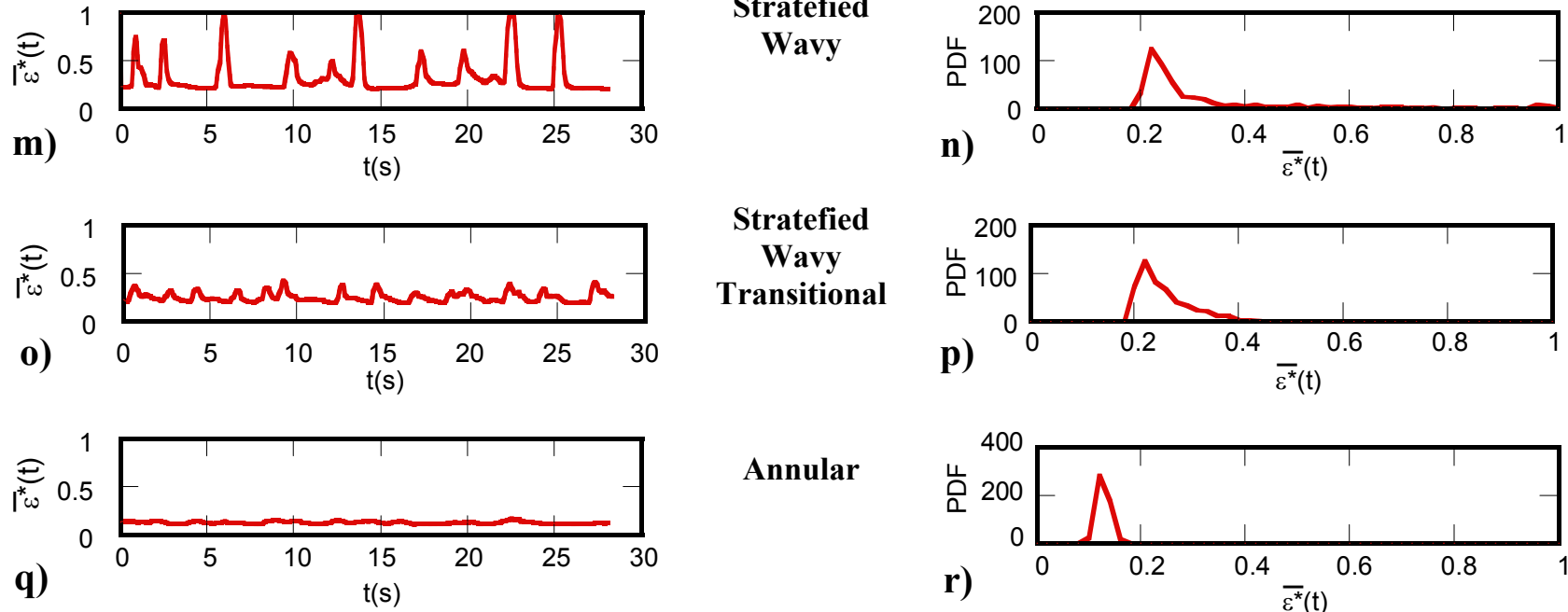

Annular

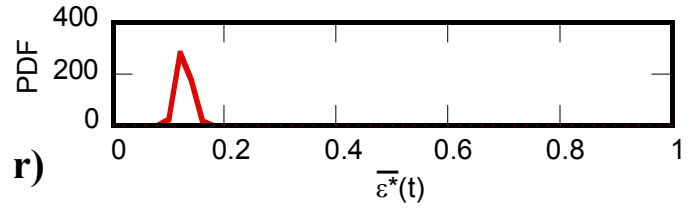

Figure 4. Plots of $\bar{g}(t)$ and $\bar{F}(t)_{R D F}$ for bubbly (a and b), bubbly transitional (c and d), plug (e and $\mathrm{f}$ ), plug transitional ( $g$ and $h$ ), slug ( $i$ and $j$ ), slug transitional ( $k$ and $l$ ), stratified wavy ( $m$ and $n)$, stratified wavy transitional ( $($ and $p$ ), and annular ( $q$ and $r)$ flows. 


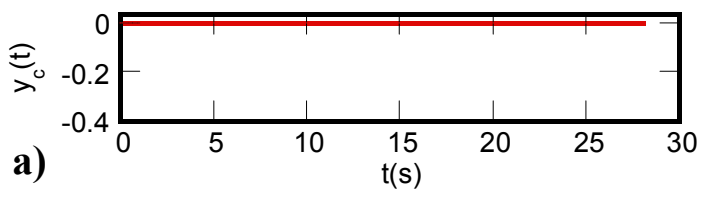

Bubbly
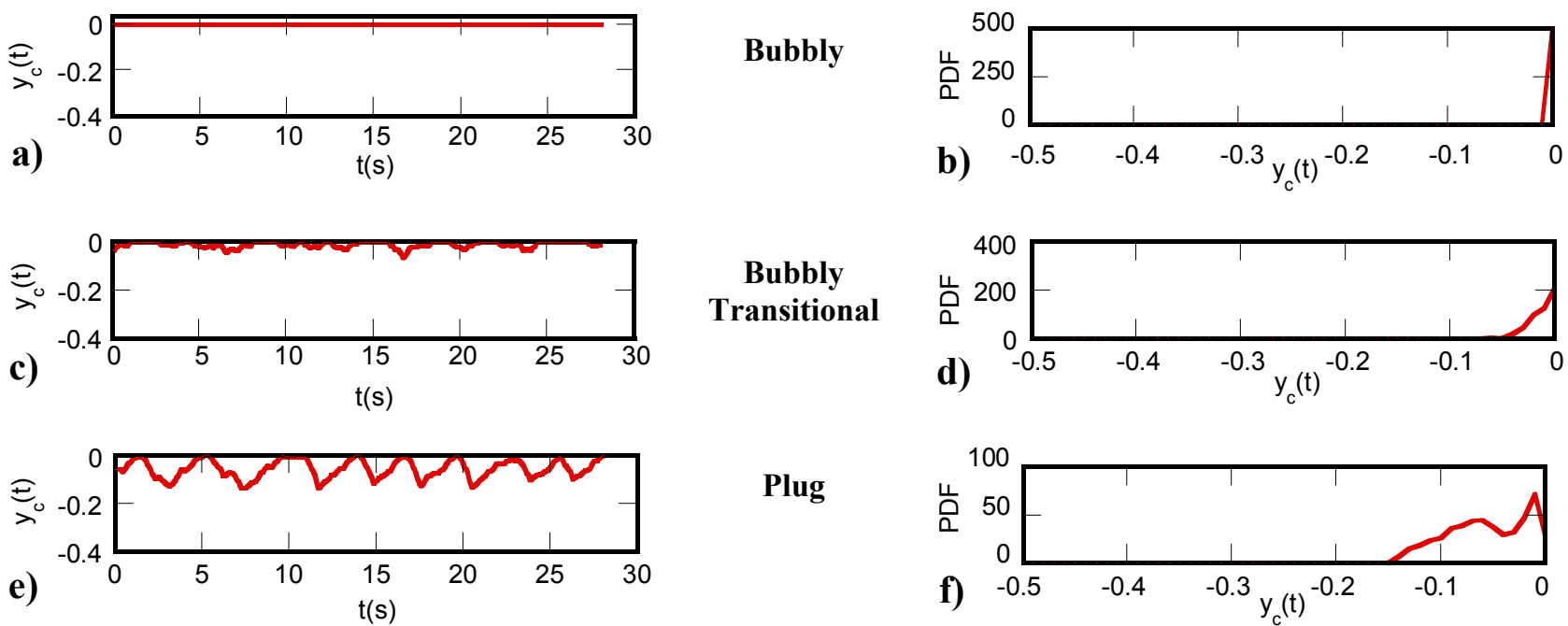

Plug
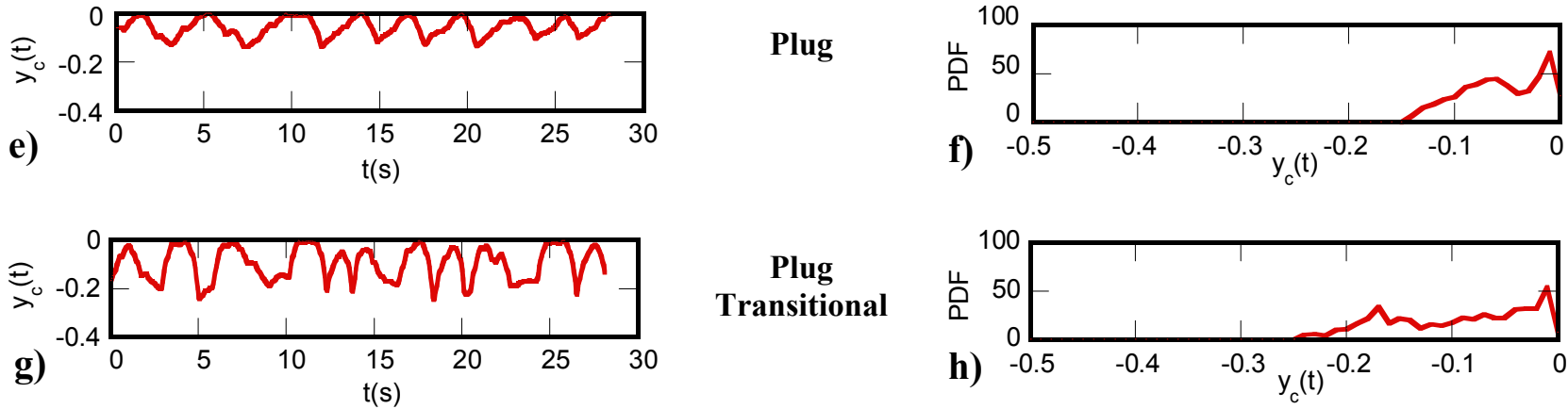

Plug

Transitional
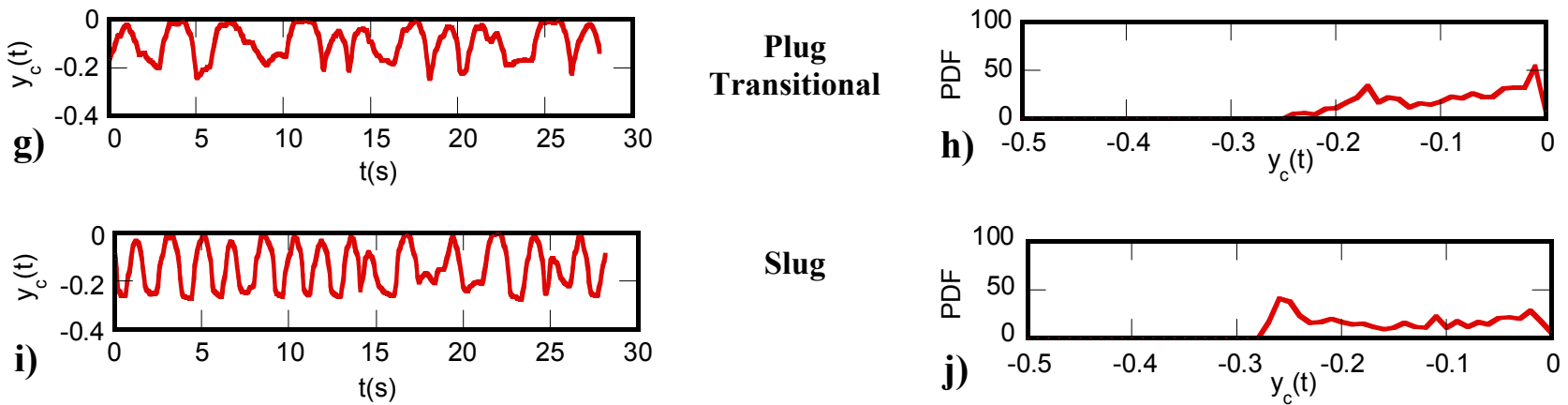

Slug
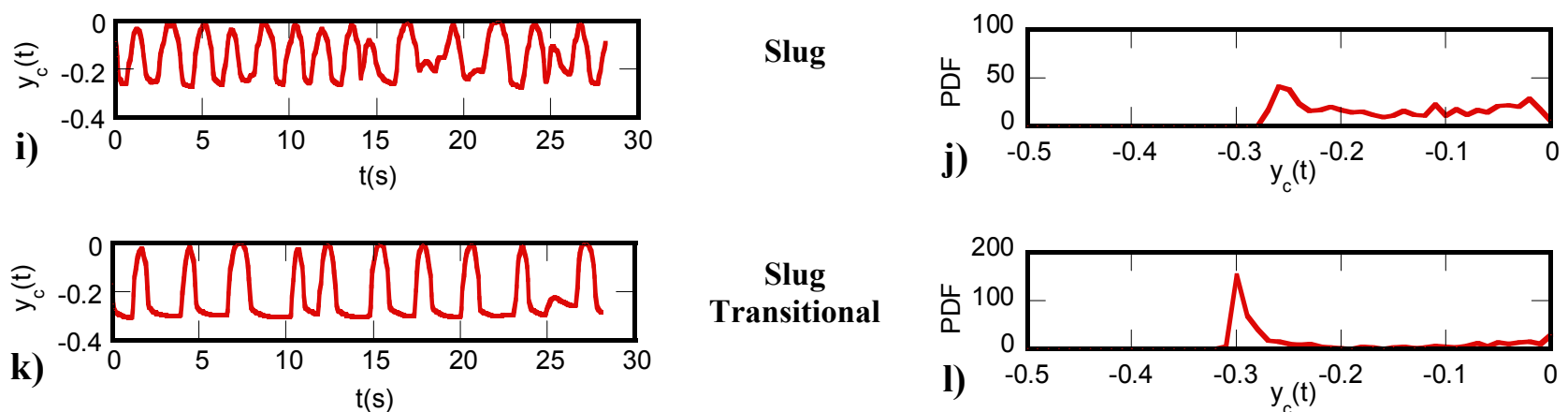

Slug

Transitional
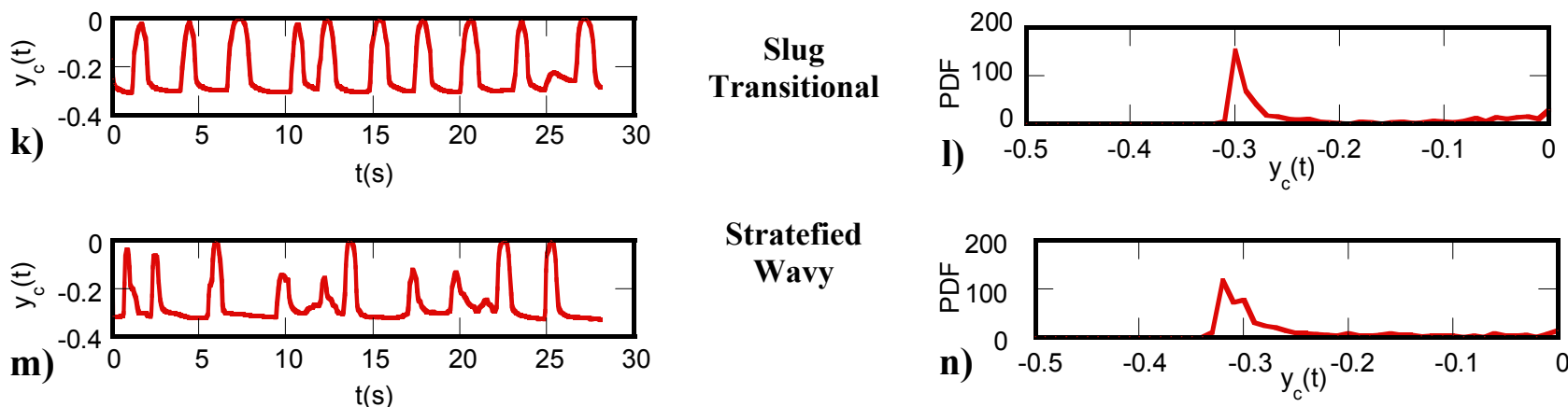

Stratefied

Wavy
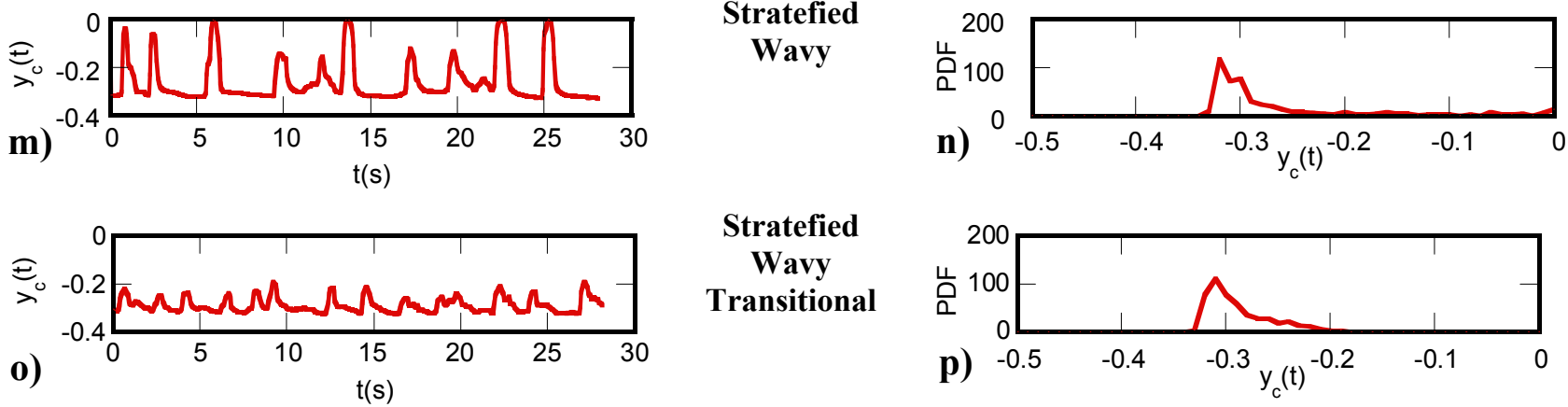

Stratefied

Wavy

Transitional
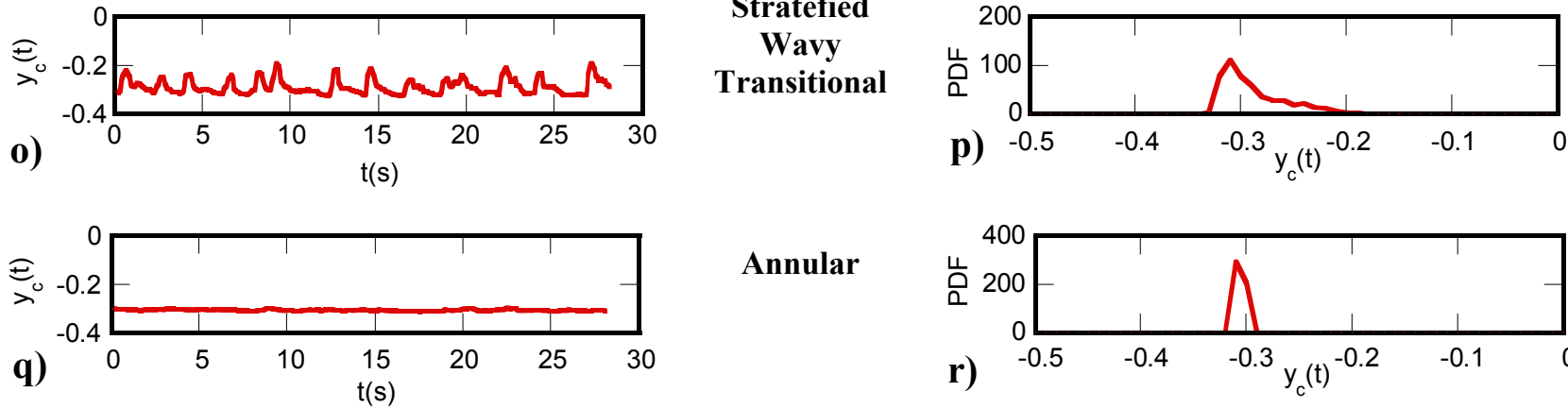

Annular

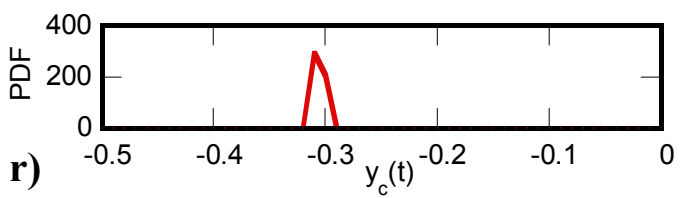

Figure 5. $y_{c}(t)$ and $y_{c}(t)_{P D F}$ behavior for bubbly (a and b), bubbly transitional (c and d), plug (e and f), plug transitional ( $g$ and $h$ ), slug ( $i$ and $j$ ), slug transitional ( $k$ and $l$ ), stratified wavy ( $m$ and $n$ ), stratified wavy transitional ( $(0$ and $p)$, and annular ( $q$ and $r$ ) flows. 
Table 1. Measurement Uncertainties

\begin{tabular}{llll}
\hline Instrument & Type & Calibration Range & Uncertainty \\
\hline Thermocouple & Type T & $5-100^{\circ} \mathrm{C}$ & $\pm 0.1^{\circ} \mathrm{C}$ \\
Pressure Transducer & Gauge & $0-1034 \mathrm{kPa}$ & $\pm 0.3 \mathrm{kPa}$ \\
Flow Meter & Turbine & $0.10-1.00 \mathrm{~L} / \mathrm{min}$ & $\pm 0.005 \mathrm{~L} / \mathrm{min}$ \\
\hline
\end{tabular}

Table 2. Summary of Two-Phase Flow Experiments

\begin{tabular}{|c|c|}
\hline Common Flow Patterns & Bubble, Plug, Slug, Strategy Wavy, Annular \\
\hline Transitional Flow Patterns & $\begin{array}{c}\text { Bubbly Transitional, Plug Transitional, Slug } \\
\text { Transitional, Stratified Wavy Transitional }\end{array}$ \\
\hline $\begin{array}{c}\text { Heater Power (W) } \\
\left.\text { Heat Flux, q" (W/cm }{ }^{2}\right)\end{array}$ & $\begin{array}{c}10-300 \\
0.40-12\end{array}$ \\
\hline $\begin{array}{c}\text { Volumetric Flow Rate } \\
(\mathrm{L} / \mathrm{min})\end{array}$ & $0.10-0.50$ \\
\hline Pressure (kPa) & $500-800$ \\
\hline Tube Diameter (mm) & 7 \\
\hline $\begin{array}{c}\text { Duration of Each Observed } \\
\text { Flow Pattern Case (s) }\end{array}$ & 30 \\
\hline
\end{tabular}


Table 3. Representative Flow Pattern Images and Characteristics

\begin{tabular}{|c|c|c|c|c|c|}
\hline $\begin{array}{c}\text { Flow } \\
\text { Pattern }\end{array}$ & $\begin{array}{c}\text { Drawing } \\
\text { of Cross } \\
\text { Section } \\
\text { Viewed } \\
\text { Along } \\
\text { Tube } \\
\text { Axis }\end{array}$ & Representative Image - Viewed Perpendicular to Tube Axis & $\begin{array}{c}\text { Time } \\
\text { Averaged } \\
\text { Void } \\
\text { Fraction } \\
\text { for Flow } \\
\text { Pattern }\end{array}$ & $\begin{array}{c}\text { Flow } \\
\text { Rate } \\
\text { (L/min) } \\
\text { for } \\
\text { Image }\end{array}$ & $\begin{array}{l}\text { Heater } \\
\text { Power } \\
\text { (W) } \\
\text { for } \\
\text { Image }\end{array}$ \\
\hline Bubbly & & - & $\begin{array}{c}0.01- \\
0.1\end{array}$ & 0.5 & 25 \\
\hline $\begin{array}{c}\text { Bubbly- } \\
\text { Transitional }\end{array}$ & & $=$ & $\begin{array}{l}0.1- \\
0.15\end{array}$ & 0.5 & 75 \\
\hline Plug & & & $0.15-0.25$ & 0.3 & 30 \\
\hline $\begin{array}{c}\text { Plug- } \\
\text { Transitional }\end{array}$ & & & $0.25-0.40$ & 0.3 & 50 \\
\hline Slug & & & $0.40-0.55$ & 0.3 & 75 \\
\hline $\begin{array}{l}\text { Slug- } \\
\text { Transitional }\end{array}$ & & $0^{\circ}$ & $0.55-0.60$ & 0.3 & 125 \\
\hline $\begin{array}{l}\text { Stratified } \\
\text { Wavy }\end{array}$ & & 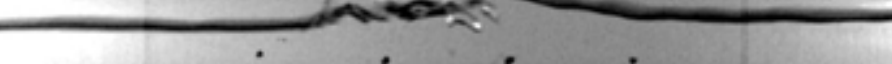 & $0.60-0.65$ & 0.3 & 180 \\
\hline $\begin{array}{c}\text { Stratified } \\
\text { Wavy- } \\
\text { Transitional }\end{array}$ & & & $0.65-0.75$ & 0.2 & 250 \\
\hline Annular & & & $0.75-0.95$ & 0.1 & 300 \\
\hline
\end{tabular}


Table 4. Comparison of Classification Success Rates Using Eight Neural Network Input Vectors for Five Basic Flow Patterns

\begin{tabular}{|c|c|c|c|c|c|c|c|c|c|}
\hline Flow Pattern & $\begin{array}{c}\text { Number } \\
\text { of } \\
\text { Runs }\end{array}$ & $\bar{s}(t)$ & $y_{Q}(t)$ & $\bar{s}(\mathbf{t})_{\mathrm{PDF}}$ & $\mathbf{y}_{\mathrm{Q}}\left(\mathbf{t}^{\prime}\right)_{\mathrm{BDF}}$ & 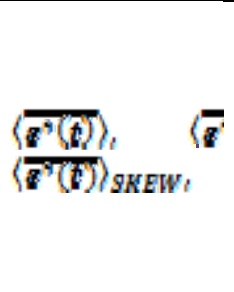 & 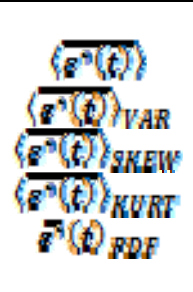 & 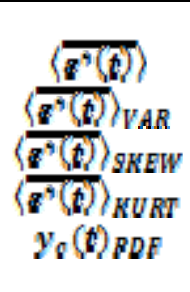 & 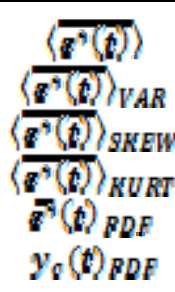 \\
\hline $\begin{array}{c}\text { Number of } \\
\text { Data in Input } \\
\text { Vector for } \\
\text { Each Run } \\
\end{array}$ & & 250 & 250 & 50 & 50 & 4 & 54 & 54 & 104 \\
\hline Bubbly & 102 & 100 & 100 & 100 & 100 & 100 & 100 & 100 & 100 \\
\hline Plug & 98 & 94.9 & 96.9 & 100 & 100 & 100 & 100 & 100 & 100 \\
\hline Slug & 129 & 86.1 & 86.1 & 98.4 & 99.2 & 100 & 99 & 100 & 100 \\
\hline St. Wavy & 129 & 94.6 & 89.1 & 100 & 100 & 99.2 & 100 & 100 & 100 \\
\hline Annular & 88 & 88.6 & 95.5 & 97.7 & 100 & 98.9 & 100 & 100 & 100 \\
\hline $\begin{array}{c}\text { Average } \\
\text { Classification } \\
\text { Success Rate } \\
\text { over All } \\
\text { Patterns }\end{array}$ & & $92.8 \%$ & $93.5 \%$ & $99.2 \%$ & $99.8 \%$ & $99.6 \%$ & $99.8 \%$ & $100 \%$ & $100 \%$ \\
\hline
\end{tabular}


Table 5. Comparison of Classification Success Rates Using Eight Neural Network Input Vectors for Nine Flow Patterns

\begin{tabular}{|c|c|c|c|c|c|c|c|c|c|}
\hline Flow Pattern & $\begin{array}{c}\text { Number } \\
\text { of } \\
\text { Runs }\end{array}$ & $\vec{s}(t)$ & $y_{0}(t)$ & $\bar{s}(t)_{B D F}$ & $y_{0}\left(t^{\prime}\right)_{\text {PDF }}$ & 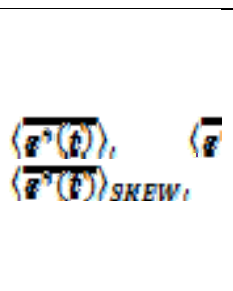 & 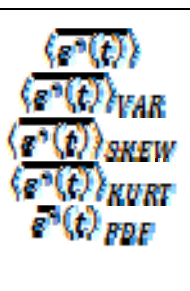 & 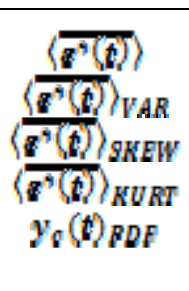 & 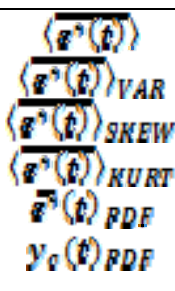 \\
\hline $\begin{array}{c}\text { Number of } \\
\text { Data in Input } \\
\text { Vector for } \\
\text { Each Run } \\
\end{array}$ & & 250 & 250 & 50 & 50 & 4 & 54 & 54 & 104 \\
\hline Bubbly & 52 & 0 & 0 & 94.2 & 94 & 94.3 & 100 & 98.1 & 100 \\
\hline Bubbly - T & 50 & 0 & 0 & 88 & 96 & 76.1 & 98 & 96.0 & 94.0 \\
\hline Plug & 58 & 32.8 & 31.1 & 100 & 97 & 100 & 100 & 98.3 & 100 \\
\hline Plug - T & 40 & 20.0 & 22.5 & 95 & 98 & 90.1 & 100 & 100 & 100 \\
\hline Slug & 89 & 29.2 & 41.6 & 80 & 79 & 94.4 & 93.3 & 92.3 & 96.6 \\
\hline Slug - T & 40 & 17.5 & 35 & 65 & 63 & 57.5 & 85 & 92.5 & 100 \\
\hline St. Wavy & 69 & 30.4 & 24.6 & 52.2 & 71 & 81.2 & 84.1 & 89.4 & 97.0 \\
\hline St. Wavy- T & 60 & 13.3 & 5 & 67 & 63 & 81.7 & 91.7 & 90.0 & 95.0 \\
\hline Annular & 88 & 39.8 & 28.4 & 95.5 & 86 & 98.9 & 97.7 & 98.9 & 100 \\
\hline $\begin{array}{c}\text { Average } \\
\text { Classification } \\
\text { Success Rate } \\
\text { over All } \\
\text { Patterns }\end{array}$ & & $20.3 \%$ & $20.9 \%$ & $81.9 \%$ & $82.9 \%$ & $86.0 \%$ & $94.4 \%$ & $95.1 \%$ & $98.1 \%$ \\
\hline
\end{tabular}

In this table, $T$ in the pattern name represents the word "transitional." 